\title{
Subunit Interactions and AMPA Receptor Desensitization
}

\author{
Antoine Robert, ${ }^{1}$ Stacey N. Irizarry, ${ }^{1}$ Thomas E. Hughes, ${ }^{2}$ and James R. Howe ${ }^{1}$ \\ Departments of ${ }^{1}$ Pharmacology and ${ }^{2}$ Ophthalmology, Yale University School of Medicine, New Haven, \\ Connecticut 06520-8066
}

\begin{abstract}
Most AMPA-type glutamate receptors (GluRs) exhibit rapid and virtually complete desensitization when activated by glutamate, and at some central synapses it is largely desensitization that determines the decay of EPSCs. However, the mechanisms underlying the conformation change that results in desensitization are not fully understood. AMPA receptor subunits that contain a single amino acid substitution have been shown to form homomeric channels that show markedly reduced desensitization. We show here that the coexpression of wild-type GluR1 with one such mutant, GluR1(L497Y), results in heteromeric channels that show desensitization behavior that is inter-
\end{abstract}

mediate between wild-type and mutant homomers. The relative amplitudes of the multiple exponential components present in the decay of glutamate-evoked currents depended on the relative abundance of wild-type and mutant subunits and were described by the combinatorial distribution of the two types of subunits into tetrameric, but not pentameric, assemblies. Our results are consistent with recent structural data suggesting that AMPA receptors are tetrameric assemblies composed of two dimers.

Key words: glutamate; AMPA receptor; desensitization; subunit interactions; allosteric; kinetics
AMPA-type glutamate receptors (GluRs) rapidly desensitize on exposure to glutamate, and the kinetics of desensitization can determine the time course of synaptic events (Jonas and Spruston, 1994; Jones and Westbrook, 1996; Otis et al., 1996). Multiple regions within single AMPA receptor subunits have been shown to influence the rate, extent, and pharmacological modulation of desensitization. Alternative splicing of the so-called flip/flop cassette has marked effects on the kinetics of desensitization (Sommer et al., 1990; Mosbacher et al., 1994), as well as on allosteric modulation by cyclothiazide and aniracetam (Partin et al., 1993, 1994, 1996). The flip and flop sequences are found in all AMPA receptor subunits and are located within the extracellular loop just before the third transmembrane segment. RNA editing at a site just upstream of the flip/flop cassette affects recovery from desensitization (Lomeli et al., 1994). Amino acids that affect desensitization are also located within the proximal portion of the extracellular loop (the S2 domain) (Mano et al., 1996). Finally, Stern-Bach et al. (1998) identified amino acids in the S1 domain that when mutated reduce the rate and extent of desensitization. Specifically, these latter authors showed that a single leucine-totyrosine substitution (L507Y for GluR3 or L497Y for GluR1) created subunits that form homomeric channels that do not appear to desensitize.

Although the above studies have identified domains within individual subunits that influence desensitization, the extent to which subunit-subunit interactions play a role is less clear. Allosteric models similar to those advanced to explain the behavior of tetrameric hemoglobin (Monod et al., 1965) have been suggested to apply to AMPA receptor desensitization (Partin et al., 1996). Such models imply, among other things, that desensitization

Received Feb. 14, 2001; revised May 9, 2001; accepted May 21, 2001.

This work was supported by National Institutes of Health Grant NS 37904. We thank Derek Bowie for providing the GluR1 constructs.

Correspondence should be addressed to James R. Howe, Department of Pharmacology, Yale University School of Medicine, 333 Cedar Street, New Haven, CT 06520-8066. E-mail: james.howe@yale.edu.

Copyright (C) 2001 Society for Neuroscience 0270-6474/01/215574-13\$15.00/0 requires a concerted conformational change involving all the subunits of the oligomeric receptor and also that agonists bind with higher affinity to the desensitized state of the receptor. Glutamate does appear to bind preferentially to the desensitized form of the receptor (Trussell and Fischbach, 1989; Patneau and Mayer, 1991; Colquhoun et al., 1992; Raman and Trussell, 1992), and evidence for a concerted conformational change comes from results showing that heteromeric assemblies of subunits with different desensitization properties display phenotypes indistinguishable from one of the "parent" subunits (Mosbacher et al., 1994; Partin et al., 1994). Recently, however, the unitary conductance of AMPA-type channels has been shown to depend on the number of ligand molecules bound to the receptor (Rosenmund et al., 1998; Smith and Howe, 2000). Because each subunit contains a single agonist binding site, one interpretation of these results is that individual subunits can gate independently.

We show here that the coexpression of wild-type GluR $1_{\text {flip }}$ with the non-desensitizing GluR1 $1_{\text {flip }}$ (L497Y) mutant results in what appears to be intermediate desensitization behavior. The relative amplitudes of the multiple components present in the decay of glutamate-evoked currents depended on the relative abundance of wild-type and mutant subunits. The results provide novel evidence that AMPA-type channels are tetrameric assemblies, and our findings are consistent with recent evidence suggesting that these assemblies are composed of two dimers.

\section{MATERIALS AND METHODS}

Cell culture. Human embryonic kidney (HEK) 293 cells were plated onto $12 \mathrm{~mm}$ glass coverslips that had been coated with poly-L-lysine $(100$ $\mu \mathrm{g} / \mathrm{ml}$ ) and were maintained in humidified $95 \% \mathrm{O}_{2} / 5 \% \mathrm{CO}_{2}$. The culture medium was modified Eagle's medium (MEM; Life Technologies) containing $10 \%$ fetal bovine serum. HEK 293 cells were transiently transfected using Lipofectamine 2000 (Life Technologies) with $0.2-0.6 \mu \mathrm{g}$ of total cDNA per coverslip. The solution used for transfection consisted of $100 \mu \mathrm{l}$ of Opti-MEM medium (Life Technologies), $3 \mu$ l of Lipofectamine $2000,0.5 \mu \mathrm{g}$ of a reporter cDNA encoding the green fluorescent protein (GFP) in pCMVsport, $0.1-1 \mu \mathrm{g}$ of GluR $1_{\text {flip }}$ or GluR $1_{\text {flip }}$ (L497Y), or both, in a CMV-driven mammalian expression vector. The GluR1 plasmids were kindly provided by Derek Bowie (Emory University, Atlanta, 
GA), who introduced the L497Y mutation. The GluR $1_{\text {flip }}$ construct (original cDNA from Peter Seeburg, MPI Medical Research, Heidelberg, Germany) was modified previously by Mark Mayer and Kathy Partin (National Institute of Child Health and Human Development, National Institutes of Health, Bethesda, MD) to enhance expression in mammalian cells. The transfection solution was first incubated at room temperature for $15 \mathrm{~min}$, and $25 \mu \mathrm{l}$ of this solution was added to each culture well. Electrophysiological recordings were performed $24 \mathrm{hr}$ after transfection.

Cerebellar culture. The cerebella of Sprague Dawley rat pups (age 5-7 d) were removed and minced with a razor blade. Cells were dissociated into the culture medium with a fire-polished Pasteur pipette in the absence of enzymes. The resulting solution was then filtered through a nylon mesh, and the cells were plated onto acid-washed coverslips coated with poly-L-lysine ( $400 \mu \mathrm{g} / \mathrm{ml}$ for $1 \mathrm{hr}$ ). The culture medium was DMEM containing $25 \mathrm{~mm} \mathrm{KCl}$ and $10 \%$ fetal bovine serum. The transfection technique was as described above. To minimize glutamate-mediated toxicity, 1,2,3,4-tetrahydro-6-nitro-2,3-dioxo-benzo[f] quinoxaline-7-sulfonamide (NBQX; RBI/Sigma) was added at $1 \mu \mathrm{M}$ to the transfected cultures.

Electrophysiology. Whole-cell patch-clamp recordings and recordings from excised outside-out patches were made with an EPC9 amplifier (HEKA). The cells were constantly superfused with normal external solution at a rate of $1 \mathrm{ml} / \mathrm{min}$. Transfected cells were identified by epifluorescence imaging of GFP (Marshall et al., 1995). Patch electrodes were pulled from thin-walled borosilicate glass with inner filament (Warner) to an open resistance of 1-3 $\mathrm{M} \Omega$. The series resistance after going whole cell was 3-6 M $\Omega$. Given the size of the peak currents in patches, the voltage errors were $\leq 5 \mathrm{mV}$ at the holding potential of -90 $\mathrm{mV}$ used for all recordings. We found that series resistance compensation $(60-80 \%)$ had no effect on the amplitude or kinetics of the glutamate-evoked currents, but did often introduce high-frequency noise; therefore it was not used in most recordings. Most currents were analog low-pass filtered at $2.9 \mathrm{kHz}$ (four-pole Bessel-type, $-3 \mathrm{~dB}$ ) and written directly to the hard-drive of the computer at a sampling rate of $30 \mathrm{kHz}$. For measurements of deactivation, the half-power frequency of the low-pass filter was set at $5 \mathrm{kHz}$, and the data were sampled at 100 $\mathrm{kHz}$. All recordings were performed at room temperature $\left(20-22^{\circ} \mathrm{C}\right)$.

The external medium was (in mM): $150 \mathrm{NaCl}, 3 \mathrm{KCl}, 2 \mathrm{CaCl}_{2}, 1$ $\mathrm{MgCl}_{2}, 5$ glucose, and 10 HEPES (pH adjusted to 7.4 with $\mathrm{NaOH}$ ). Pipettes were filled with a solution containing (in mM): $120 \mathrm{CsF}, 33$ $\mathrm{KOH}, 2 \mathrm{MgCl}_{2}, 1 \mathrm{CaCl}_{2}, 0.1$ spermine, 10 HEPES, and 11 EGTA (pH adjusted to 7.4 with $\mathrm{CsOH}$ ). Glutamate was added to the external solution. Cyclothiazide was prepared in DMSO and diluted in external solution to a final concentration of $100 \mu \mathrm{M}$ (final DMSO concentration $0.5 \%$ ). All chemicals were purchased from Sigma (St. Louis, MO).

Fast perfusion. In most experiments, glutamate was applied with a rapid perfusion system made from a pulled theta capillary. The tip of the theta glass was cleanly broken with a diamond cutter to a tip diameter of $\sim 300 \mu \mathrm{m}$, and its septum was snapped back at the tip with a sharp needle so that the flow of solution leaving the tip from either side of the theta capillary overlapped. Four capillaries (outer diameter $450 \mu \mathrm{m}$; Polymicro Technologies) were introduced into each barrel at the back of the theta glass and secured with Sylgard (Dow Corning). Each capillary was connected to plastic tubing that branched to a solenoid valve (The Lee Company). The valves were opened and closed by a analog-to-digital (A/D) interface (TIB 14, HEKA) that was controlled by the acquisition software of the EPC9. The speed of the solution exchanges obtained with this system was estimated with an open patch pipette by measuring the current deflections induced by a change in the $\mathrm{NaCl}$ concentration. The mean $10-90 \%$ rise times of the open-tip responses were $275 \pm 25 \mu$ sec $(n=5)$.

The valve-controlled system described above produced rapid solution exchanges, but it did not allow us to make applications briefer than $\sim 50$ msec. For measurements of deactivation time constants, and for experiments to determine the effect of cyclothiazide on peak currents, glutamate was applied to excised patches using a theta glass pipette mounted on a piezoelectric bimorph. The theta glass pipettes were pulled and broken to a tip diameter of $\sim 300 \mu \mathrm{m}$. The width of the septum separating the two barrels was reduced by etching with hydrofluoric acid to increase the sharpness of the interface created between solutions flowing out of each barrel. Patches were positioned near the solution interface, and the interface was moved by applying voltage across the bimorph with a constant voltage source (Winston Electronics Co.) that was triggered with one of the A/D outputs on the EPC9. The voltage pulses were resistance-capacitive filtered to minimize mechanical oscillations of the piezoelectric device. The $10-90 \%$ rise times of the open-tip responses obtained with this system were 100-200 $\mu$ sec, and glutamate applications as brief as $1 \mathrm{msec}$ could be delivered reproducibly.

Data analysis. The digitized records were transferred to IGOR software (Wavemetrics). The decays of glutamate-evoked currents were fitted with functions consisting of the mixture of multiple exponential components and a steady-state plateau current. Usually 5-10 responses were averaged, and the fits were performed on the averaged currents. The segment of the decay to be fitted was defined by placing cursors on the data trace, where the first cursor was placed near the peak of the current and the other cursor $\sim 700 \mathrm{msec}$ after the peak. Fits were performed by sequentially including from one to four exponential components. At each stage, the quality of the fit was evaluated by visual inspection of the residual current (obtained by subtracting the fit from the data). If the residual current was not flat, an additional exponential component was added. Two exponential components and a plateau current gave adequate fits in a few cases, and functions containing three exponentials were sufficient to obtain a flat residual current in all cases. Not only did the inclusion of a fourth component not improve the fits as assessed by visual inspection of the residual currents, but repeated four-component fits of the same data gave parameter values that were not reproducible. In most cases, the amplitude of one component converged to a value near zero, two of the time constants converged to very similar values, or one of the time constants converged to a value that was very brief or very long. Inclusion of a fourth component typically reduced the sum of squared deviations by $<1 \%$ and rarely improved the goodness of fit significantly as assessed by a standard test for comparison of nested models (Horn, 1987). In contrast, repeated three-exponential fits of the same decays gave reproducible time constants and relative amplitudes, and the parameter values obtained were largely insensitive to the initial guesses. With the exception of some of the results obtained at mutant/ wild-type ratios of $1: 6$ and $3: 1$, the three-exponential fits were significantly better than those obtained with two-exponential components.

The recovery from desensitization was estimated with two-pulse protocols in which a constant $100 \mathrm{msec}$ application of $500 \mu \mathrm{M}$ glutamate was followed by test pulses applied at variable intervals. Each two-pulse protocol was separated by a $5 \mathrm{sec}$ interval to allow complete recovery from desensitization. The decays of the currents evoked by the test pulses were fitted as described above, and the amplitudes of the fast and intermediate exponential components were plotted as a function of recovery time. Each set of data was fitted with a single exponential function: $I_{\mathrm{t}} / I_{\infty}=1-\exp \left((t-o f f s e t) / \tau_{\text {rec }}\right)$, where $I_{\mathrm{t}}$ is the amplitude of the current for a given test pulse, $I_{\infty}$ is the amplitude of the corresponding component for the current evoked by the constant prepulse application, $t$ is the interpulse interval, and $\tau_{\text {rec }}$ is the time constant of the exponential recovery. The single exponential fits to the recovery data did not extrapolate back to zero time. Thus the additional variable, offset, was included in the fits and subtracted from all recovery times. This offset may reflect slight delays in the complete removal of glutamate after switching back to control solution at the end of the prepulse application. To minimize this offset, we used $500 \mu \mathrm{M}$ glutamate for the recovery experiments instead of the higher concentration ( $5 \mathrm{~mm}$ ) used in most experiments.

Fluctuation analysis of steady-state currents evoked by a range of glutamate concentrations was used to estimate the unitary conductance and open probability of GluR $1_{\text {flip }}$ (L497Y) homomeric channels. These results were compared with the corresponding results obtained for wildtype GluR $1_{\text {flip }}$ channels in the presence of $100 \mu \mathrm{M}$ cyclothiazide (to slow desensitization). Values for the current variance were obtained from spectral density analysis of steady-state currents evoked at different glutamate concentrations, and these values were plotted against the corresponding mean currents to give estimates of $P_{\text {open }}$ and the apparent unitary conductance $\left(\gamma_{\text {noise }}\right)$. For these experiments, the data were sampled at $9.4 \mathrm{kHz}$ and low-pass filtered at $2 \mathrm{kHz}$. Power spectra were obtained and fitted with bi-Lorentzian functions as described previously (Howe, 1996).

It was assumed that the assembly of wild-type and mutant subunits was random and combinatorial. The probabilities for each possible stoichiometric combination of wild-type and mutant subunits were calculated from the binomial relationship: $P_{\mathrm{j}}=(N ! /(j !(N-j) !))\left(p_{\text {mut }}\right) j(1-$ $\left.p_{\text {mut }}\right)^{N-j}$, where $P_{\mathrm{j}}$ is the probability that a channel composed of $N$ subunits will contain $j$ mutant subunits, and $p_{\text {mut }}$ is the amount of GluR1 $_{\text {flip }}$ (L497Y) cDNA as a proportion of the total GluR-encoding cDNA (wild type plus mutant) used for the transfection. For each wild-type/mutant cDNA ratio studied, the results were compared with the stoichiometric predictions for channels containing $N=4$ and $N=5$ subunits (tetramers and pentamers, respectively). Similar calculations 
were done, assuming that the channels assembled first as dimers, to obtain the probabilities that a channel contained at least one dimer in which both subunits were the L497Y mutant.

Simulated glutamate-evoked currents were generated using QUB software (Qin et al., 1996, 1997). The kinetics of the simulated currents were analyzed as described above in IGOR. The rate constants and parameter values used for the simulations are given in Results or in the legends to the appropriate figures. The experimental results in Figures $5 C, 6 \mathrm{~A}$, and $7 B$ are shown as mean $\pm \mathrm{SD}$; all other results are given as mean $\pm \mathrm{SEM}$.

\section{RESULTS}

\section{Desensitization of GluR1 flip $_{\text {and GluR1 flip }}$ (L497Y) homomeric channels}

The transient transfection of HEK 293 cells with the cDNAs encoding the GluR $1_{\text {flip }}$ and the GluR $1_{\text {flip }}$ (L497Y) subunits resulted in high-level expression of each type of homomeric channel. This facilitated our studies of desensitization kinetics because we were able to measure large ensemble currents in outside-out patches from transfected cells. Although the superfusion systems used produced solution exchanges of $<300 \mu \mathrm{sec}$, the rise times of whole-cell currents were much slower (2-5 msec), presumably because of diffusion barriers. Therefore, although qualitatively similar data were obtained from whole-cell recordings, all quantitative analysis reported here was limited to results obtained in outside-out patches in which the rise times of the currents were $<500 \mu$ sec.

Typical currents evoked by $5 \mathrm{~mm}$ glutamate in outside-out patches are shown in Figure 1. Currents through wild-type GluR $1_{\text {flip }}$ homomers showed rapid and virtually complete desensitization. Although the decay of the currents proceeded largely monophasically, we found that these decays were consistently better fitted by two, rather than one, exponential component. Figure $1 A a$ shows the current evoked by the sustained application of $5 \mathrm{~mm}$ glutamate in a patch from a GluR $1_{\text {flip }}$-transfected cell. The two-exponential fit is superposed on the data, and the corresponding residual current is also shown. An expanded view of the same current with the best single exponential fit superposed is shown in Figure $1 \mathrm{Ab}$. As was the case in all patches studied, single exponential fits failed to describe well the tail of the glutamate-evoked currents and also gave significant residual current near the peak response. The source of the small slower component is uncertain, but we believe it arose because a few of the receptors in the patches had restricted access to the agonist because of physical barriers to diffusion. To limit the effect of this small tail on the time constants measured for the major component of decay, the slow component was routinely included in the fitting. On average, the currents through $\mathrm{GluR} 1_{\text {flip }}$ homomers decayed with a large fast component $\left(\tau_{\mathrm{f}}=2.24 \pm 0.12 \mathrm{msec}\right.$; $98-99.5 \%$ of the total current amplitude; $n=8$ patches) and a much smaller component with a time constant that varied between 9 and $22 \mathrm{msec}$. The sustained component of the currents was very small, averaging only $0.23 \pm 0.09 \%$ of the total peak amplitude $(n=8)$.

Typical currents evoked by glutamate pulses in patches from cells expressing GluR1 flip (L497Y) channels are illustrated in Figure $1 B$. As shown by Stern-Bach et al. (1998), these channels generate a sustained current that does not show the rapid decay that is characteristic of wild-type AMPA receptor-mediated currents. However, in each of the seven patches analyzed here, the glutamate-activated currents did show some decay, albeit slow and incomplete (Fig. $1 \mathrm{Ba}$ ). Single exponential fits to these decays gave a mean time constant of $111 \pm 13 \mathrm{msec}$; the mean amplitude of the sustained plateau current was $86 \pm 2 \%$ of the peak

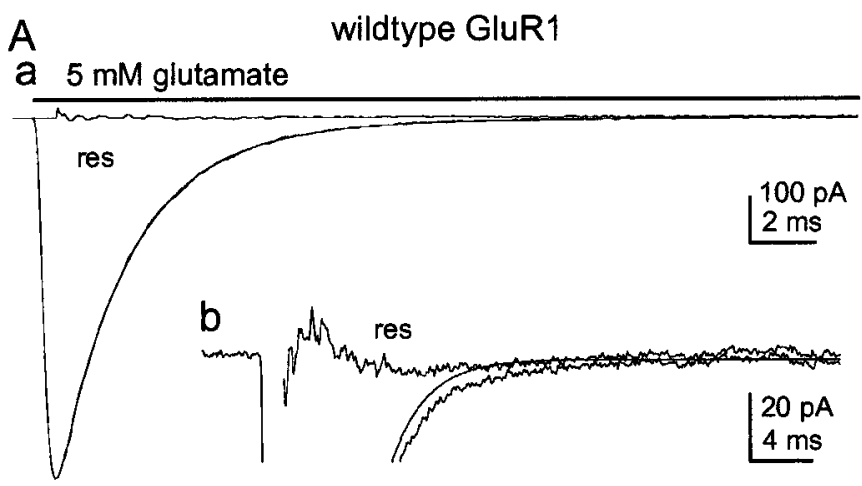

B GluR1 (L497Y)
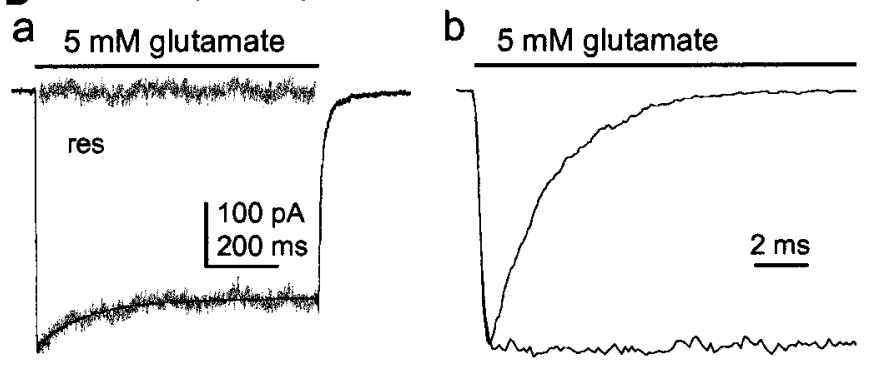

Figure 1. Desensitization kinetics of wild-type GluR $1_{\text {flip }}$ and GluR $1_{\text {flip }}$ (L497Y). Aa, Current elicited by the rapid and sustained application of 5 mM glutamate (bar) in an outside-out patch from a HEK 293 cell transfected with wild-type GluR $1_{\text {flip }}$. The bi-exponential fit (smooth solid line) to the decay of the current, as well as the residual trace (res; obtained by subtracting the recording from the fit), are shown. The fit gave time constants of 2.25 and $14 \mathrm{msec}$ for the two components. The amplitude of the fast component was $99 \%$ of the total peak current. $A b$, Enlargement of the same current fitted with a single exponential. Note that the fit is poor in the tail of the current, as well as the presence of substantial residual current near the peak. $\mathrm{Ba}$, Response to $5 \mathrm{~mm}$ glutamate in a patch from a cell expressing GluR $1_{\text {flip }}(\mathrm{L} 497 \mathrm{Y})$. The single-exponential fit to the decay and the corresponding residual current are shown. $B b$, The rises of

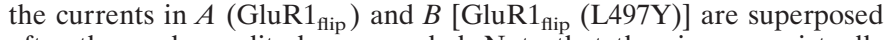
after the peak amplitudes are scaled. Note that the rises are virtually identical.

amplitude $(n=7)$. It is therefore likely that some residual desensitization is still present in the mutant channels.

The L497Y mutation did not appear to affect the speed of activation. The currents in Figure $1, A$ and $B a$, have been normalized and superposed in Figure $1 B b$ to illustrate the similar rise of the currents through wild-type and mutant channels.

\section{Heteromeric channels display intermediate desensitization kinetics}

To determine the relative dominance of the wild-type and mutant phenotypes, we recorded currents activated by $5 \mathrm{~mm}$ glutamate in outside-out patches from HEK 293 cells that were cotransfected with equal amounts of the plasmids encoding GluR $1_{\text {flip }}$ and $\mathrm{GluR}_{\text {flip }}$ (L497Y). A typical example of the recordings obtained in these experiments is presented in Figure 2. In each of six patches, three exponential components were required to obtain adequate fits to the decays of the glutamate-activated currents. The three individual components are shown in Figure $2 B$ as dotted lines. The mean values obtained for the time constant of the fast $\left(\tau_{\mathrm{f}}\right)$, intermediate $\left(\tau_{\mathrm{i}}\right)$, and slow $\left(\tau_{\mathrm{s}}\right)$ components were $3.94 \pm 0.14,19.5 \pm 1.2$, and $94 \pm 10 \mathrm{msec}$, with respective 


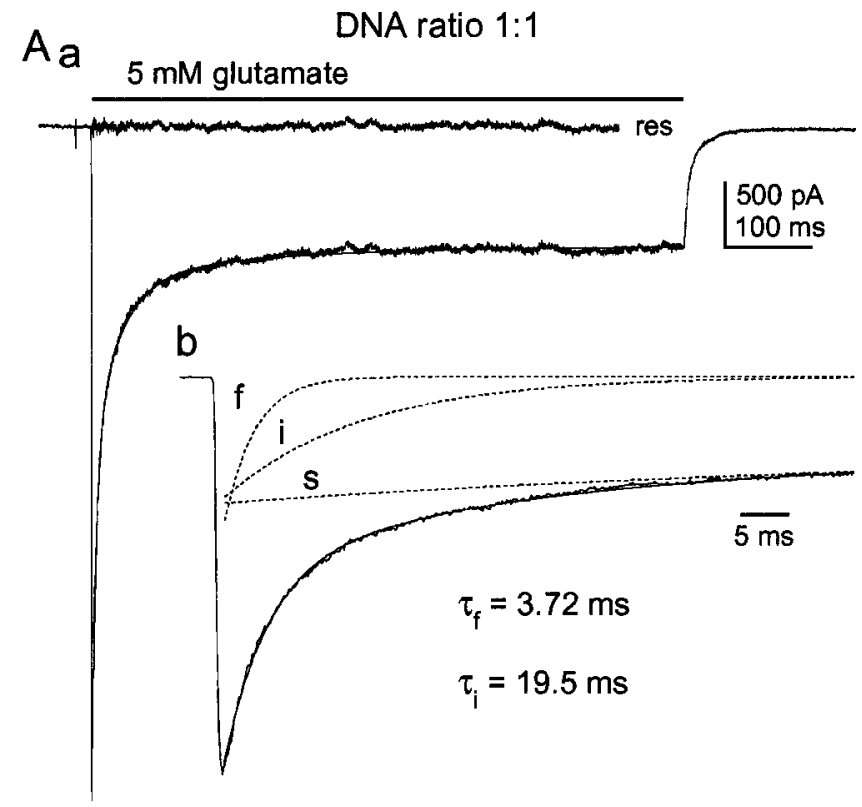

Figure 2. Coexpression of GluR $1_{\text {flip }}$ and GluR $1_{\text {flip }}$ (L497Y) gives an intermediate component of decay. $A a$, Current elicited by $5 \mathrm{~mm}$ glutamate (bar) in a patch from a cell transiently cotransfected with equal amounts of wild-type GluR1 $1_{\text {flip }}$ and the GluR $1_{\text {flip }}$ (L497Y) mutant. The decay of the current contains three resolvable exponential components. The triple exponential fit (smooth solid line) and the corresponding residual current (res) are shown. $A b$, The same current and the fit are shown on an expanded time scale, together with each of the individual exponential components (dotted lines). The three components decay at fast $\left(f ; \tau_{\mathrm{f}}=\right.$ $3.72 \mathrm{msec})$, intermediate $\left(i ; \tau_{\mathrm{i}}=19.5 \mathrm{msec}\right)$, and slow $\left(s ; \tau_{\mathrm{s}}=97 \mathrm{msec}\right)$ rates. The slow component of decay and the steady-state plateau current are treated as a single component. Each of the three components represents approximately one-third of the total current.

amplitudes of $31 \pm 1.6,30 \pm 1.2$, and $12 \pm 1.6 \%$ of the total peak current ( $n=6$ patches). At this 1:1 DNA ratio, the sustained current represented $27 \pm 2.1 \%$ of the total current. Because the slow component decayed with a time constant that was indistinguishable from that of homomeric GluR1 $1_{\text {flip }}$ (L497Y) channels (Fig. $1 B a$ ), the slowly decaying component and the sustained current were treated as a single desensitization phenotype, which is hereafter referred to as the slow component.

The results of these coexpression experiments indicated that the channel population generated by mixing wild-type and nondesensitizing subunits displays three distinct phenotypes that are characterized by different rates and extents of desensitization. Under conditions in which the expression of the two types of subunits is approximately equal (1:1 cDNA ratio), the three components that are distinguishable in the decay of large ensemble currents have similar amplitudes. Approximately one-third of the channels desensitize with a rapid time course that is slightly slower than that of homomeric wild-type channels, approximately one-third desensitize at a rate $\left(\tau_{\mathrm{i}} \approx 20 \mathrm{msec}\right)$ that is intermediate between wild-type and mutant homomers, and the remaining one-third desensitize incompletely and at a rate that is similar to that of homomeric mutant channels.

\section{The amplitude of each phenotype depends on the ratio} of wild-type and mutant cDNAs

One interpretation of the results obtained in the coexpression experiments is that the rate and extent of desensitization depends on the stoichiometry of wild-type and mutant subunits in individ- ual channel assemblies. To investigate this possibility further, we tested different mutant/wild-type cDNA ratios to vary the relative abundance of mutant and wild-type subunits. With the exception of the L497Y mutation, the expression plasmids used in our experiments were identical. Figure 3 shows typical results obtained in outside-out patches from cells transfected with cDNA mixtures at mutant/wild-type ratios of 1:6 $(A), 1: 3(B)$, and 3:1 $(C)$. Each panel of Figure 3 shows the current evoked by a 700 msec application of $5 \mathrm{~mm}$ glutamate, the fit to its decay, and the corresponding residual current. The insets show the same recordings on an expanded time scale with the individual components (fast, intermediate, and slow) that were detected in the decays shown as dotted lines.

Three main findings were evident from the coexpression experiments. First, as the mutant/wild-type cDNA ratio was systematically increased, the relative amplitude of the fast component of decay decreased and the relative amplitude of the slow component increased. At a mutant/wild-type ratio of 3:1, the fast component was very small or absent. Second, concomitant with these changes, the time constant of the fast component became gradually slower. Third, at each cDNA ratio tested, there was a component of decay that proceeded with a time constant of $\sim 20$ msec. The fits to the currents evoked in patches from cells transfected with mutant and wild-type cDNAs at a ratio of 1:6 gave mean $\tau_{\mathrm{f}}$ and $\tau_{\mathrm{i}}$ values of $3.04 \pm 0.08$ and $22.3 \pm 1.6 \mathrm{msec}$, respectively $(n=7)$. The relative amplitudes (as a proportion of the total peak current) were $0.90 \pm 0.02$ for the fast component, $0.08 \pm 0.01$ for the intermediate component, and $0.02 \pm 0.004$ for the slow component. In the experiments in which a 1:3 mutant/ wild-type ratio was used, we found $\tau_{\mathrm{f}}=3.52 \pm 0.10, \tau_{\mathrm{i}}=20.2 \pm$ 1.6 , and $\tau_{\mathrm{s}}=117 \pm 10 \mathrm{msec}(n=5)$. The relative amplitudes were $0.66 \pm 0.04$ for the fast component of decay, $0.17 \pm 0.02$ for the intermediate component, and $0.085 \pm 0.001$ for the slow plus sustained current. The results from the experiments using a mutant/wild-type ratio of 3:1 gave mean $\tau_{\mathrm{i}}$ and $\tau_{\mathrm{s}}$ values of $18.4 \pm$ 1.2 and $121 \pm 8 \mathrm{msec}$, respectively $(n=5)$. The relative amplitudes of the intermediate and slow components were $0.19 \pm 0.02$ and $0.81 \pm 0.02$.

\section{Comparison of the results with combinatorial predictions}

The results above show that the relative amplitudes of the three components distinguished in the decay of currents evoked by sustained applications of glutamate changed in a graded fashion as the mutant/wild-type cDNA ratio was altered. To test further the hypothesis that the three components arise from channels with different stoichiometries of mutant and wild-type subunits, we calculated the proportions of each possible subunit combination, assuming that channel assembly is combinatorial.

The combinatorial analysis that we performed rests on several simplifying assumptions. First, we assumed that the relative abundance of wild-type and mutant subunits accurately reflects the ratio of the two cDNAs used for the transfection. Because the expression levels were high, and the two cDNA constructs were identical except for the point mutation, we believe that this assumption is reasonable. Second, our analysis assumed that the point mutation does not affect subunit assembly. Although this assumption is untested, the levels of expression seen when each cDNA was expressed alone were similar, and some channel properties, e.g., the apparent rate of activation, were unaltered by the mutation.

The combinatorial analysis also assumes that the unitary con- 

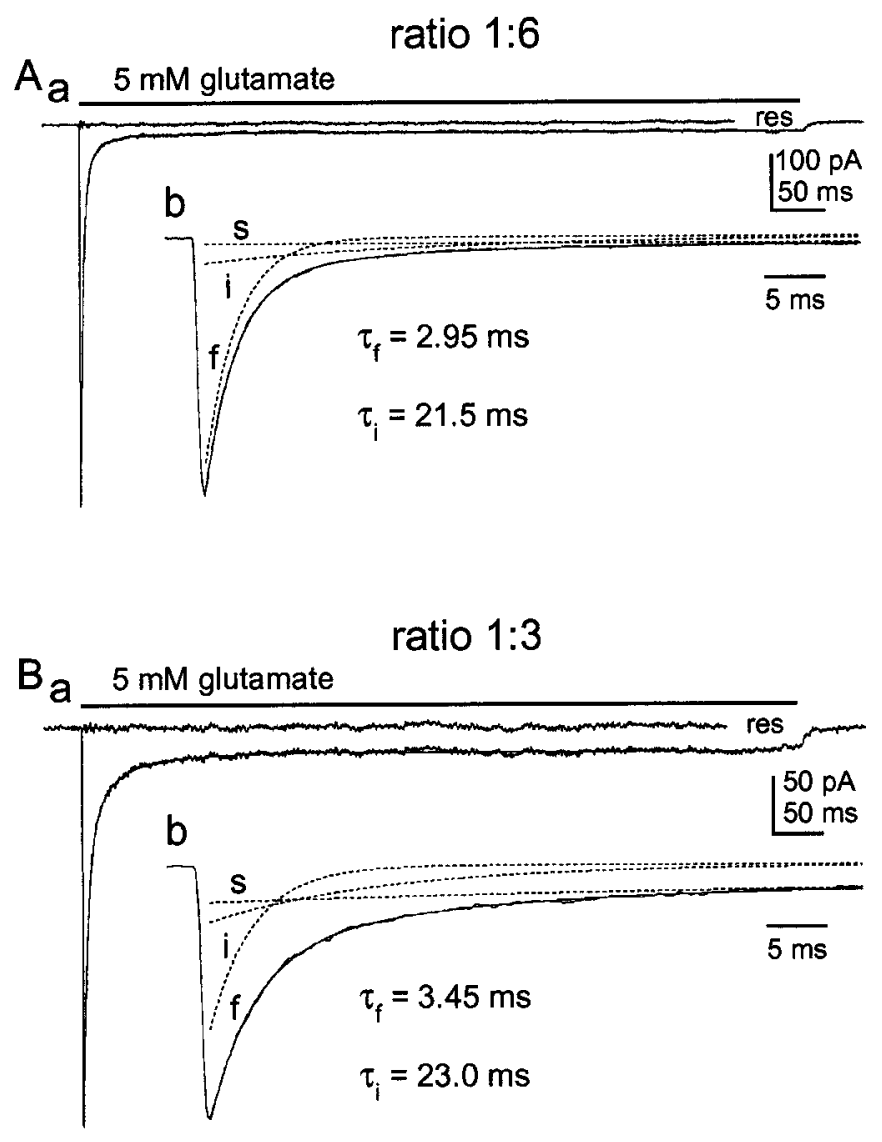

\section{ratio $3: 1$}

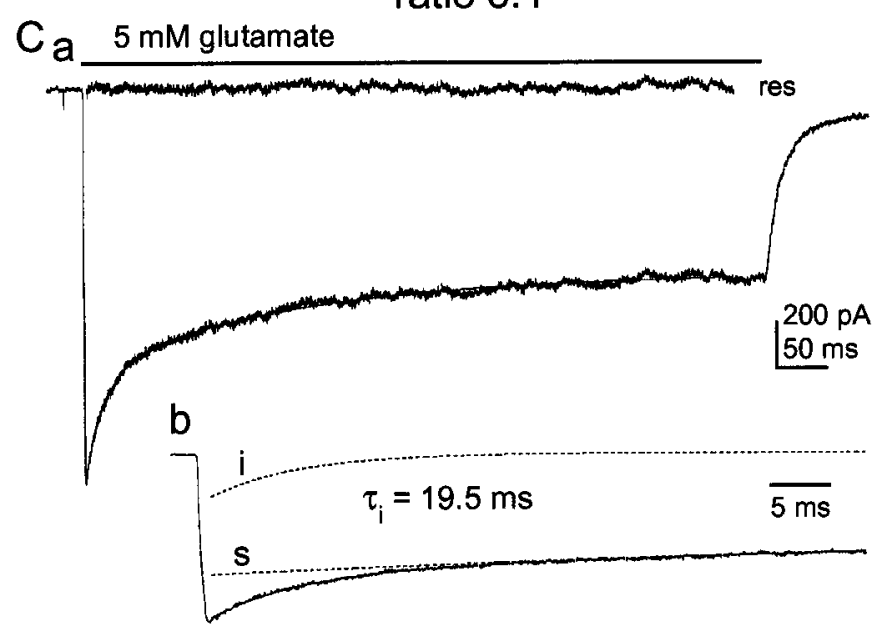

Figure 3. The relative amplitudes of the fast, intermediate, and slow components of decay vary systematically with the ratio of wild-type and mutant cDNAs. $A a$, Glutamate-evoked current in a patch from a cell cotransfected with plasmids encoding GluR $1_{\text {flip }}$ (L497Y) and GluR $1_{\text {flip }}$ at a cDNA ratio of 1:6. The decay of the current was fitted with two exponential components and a small sustained current. The fit (smooth solid line) and the residual current (res) are shown. $A b$, Same trace as in $A a$ on an enlarged time scale together with the three individual components (dotted lines). The values for $\tau_{\mathrm{f}}$ and $\tau_{\mathrm{i}}$ are given. $B$, Same as $A$, except that the cell from which the patch was excised was cotransfected with GluR $1_{\text {flip }}(\mathrm{L} 497 \mathrm{Y})$ and GluR $1_{\text {flip }}$ cDNAs at a ratio of 1:3. $C$, Same as $A$, except that the transfection mixture contained GluR1 $1_{\text {flip }}$ (L497Y) and GluR $1_{\text {flip }}$ cDNAs at a ratio of 3:1. Note that there is no fast component of decay. The time constants obtained for the fast and intermediate components are given in each panel. ductance and probability of channel opening were similar for wild-type and mutant channels. It has been shown that the corresponding leucine-to-tyrosine mutation in GluR3 (L507Y) does not alter either the main conductance level or the open probability $\left(P_{\text {open }}\right)$ of homomeric channels formed from this subunit (Rosenmund et al., 1998; Stern-Bach et al., 1998). We performed fluctuation analysis of steady-state currents over a range of five to seven glutamate concentrations to estimate the unitary conductance and $P_{\text {open }}$ of homomeric GluR1 $1_{\text {flip }}$ (L497Y) channels. These results were compared with the corresponding results obtained for wild-type GluR $1_{\text {flip }}$ channels in the presence of $100 \mu \mathrm{M}$ cyclothiazide (to slow desensitization). At saturating agonist concentrations, the $\gamma_{\text {noise }}$ values obtained for both types of homomeric channels were similar (wild-type GluR1: $24.9 \pm 1.7 \mathrm{pS}, n=$ 7; GluR1 (L497Y): $24.6 \pm 1.9 \mathrm{pS}, n=4)$, and the $P_{\text {open }}$ was 0.8-0.9. These $P_{\text {open }}$ values are similar to the corresponding values for the peak $P_{\text {open }}$ of wild-type GluR1 channels with intact desensitization (Banke et al., 2000).

Finally, our combinatorial analysis implicitly assumed that the channels in the excised patches are representative of the population expressed in a given cell. The patches used for the analysis gave peak inward currents that were always $>300 \mathrm{pA}$ and were typically $\sim 1 \mathrm{nA}$ (at the routine holding potential of $-90 \mathrm{mV}$ ). Given the unitary conductance of GluR1 channels (Derkach et al., 1999; Banke et al., 2000; this paper), the peak currents all correspond to more (usually much more) than 150 channels open simultaneously, which is a sufficient number to be representative.

With the above assumptions, the probability of each possible wild-type/mutant stoichiometry can be predicted from the binomial equation (see Materials and Methods). The calculated probabilities for the possible wild-type and mutant subunit combinations are given in Figure 4 for each of the four mutant/wild-type cDNA ratios investigated. Figure $4 A$ gives the combinatorial predictions assuming that the receptor is a tetramer, whereas the probabilities in Figure $4 B$ assume the channel is a pentamer. These probabilities can then be compared with the relative amplitudes of the three exponential components distinguished in the current decays. If AMPA receptors are tetramers, the results obtained with a 1:1 cDNA ratio (approximately equal amounts of fast, intermediate, and slow current) can be accounted for if the rapidly decaying component corresponds to channels containing three or four wild-type subunits $(0.25+0.06=0.31)$, the intermediate component corresponds to channels with two wild-type and two mutant subunits [0.37 (Fig. 4A, shaded row)], and the slow component arises from channels with three or four mutant subunits $(0.06+0.25=0.31)$. This partitioning of the probabilities also matches well our observation that the relative amplitude of the intermediate component is similar for mutant/wild-type ratios of $1: 3$ and $3: 1$ ( 0.17 and 0.19 vs 0.21 predicted). The relative amplitude of the intermediate component at a mutant/wild-type ratio of 1:6 also agrees with the predictions ( 0.08 observed vs 0.09 predicted).

In contrast to the results obtained assuming the channel is a tetramer, there is no partitioning of the probabilities in Figure $4 B$ (channels assumed to be pentamers) that matches well the relative amplitudes of the three components over the range of cDNA ratios tested. This is so despite the fact that there are more ways to partition the results, which might be expected to increase the chance of a spurious match. In particular, there is no partitioning that can account for the roughly equal amplitudes of each component at a cDNA ratio of $1: 1$, nor our observation that the amplitude of the intermediate component was similar at mutant/ 

Probability of each tetrameric combination

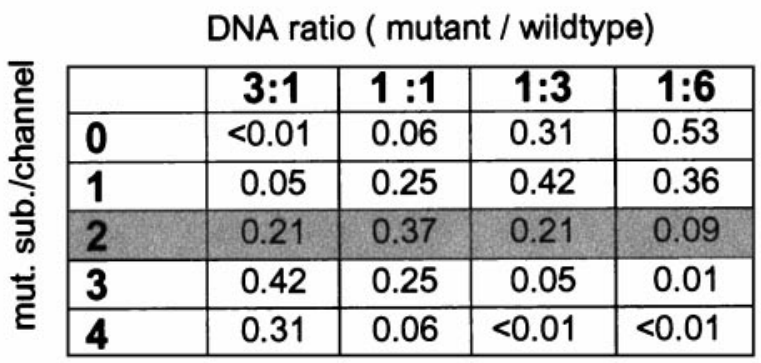

B

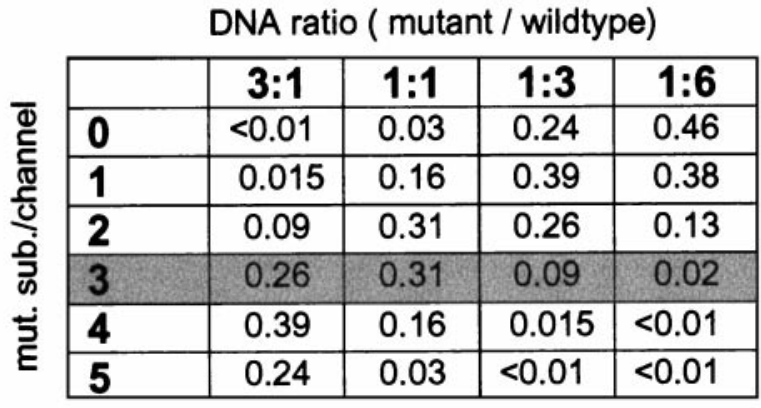

Figure 4. The results support the idea that AMPA receptors are tetramers. $A$, Table showing the probability of occurrence of each possible subunit combination if the channels are tetramers for the different cDNA ratios examined. Each row corresponds to channels with a different number of mutant subunits $(0,1,2,3,4)$ as indicated. The shaded row corresponds to channels containing two mutant subunits and predicts well the measured relative amplitude of the intermediate component. The relative proportions of the fast component of desensitization match well the predicted proportion of channels containing no or one mutant subunit. The relative proportions of the slow component match well with the sum of homomeric mutants and channels containing one wild-type subunit. $B$, Probability of occurrence of all subunit combinations if the channels are pentamers. No partitioning of the subunit combinations matches the proportion of fast, intermediate, and slow phenotypes over the range of cDNA ratios tested.

wild-type ratios of $1: 3$ and 3:1. For example, if the intermediate component is identified with channel assemblies containing three mutant subunits [(Fig. 4B, shaded row) 0.31 probability at a 1:1 ratio], then it is predicted that the fast component should be more than twice the amplitude of the slow component ( 0.5 vs 0.19 ). This way of partitioning the pentameric probabilities also predicts that the intermediate component should have been very small (0.02) at a mutant/wild-type ratio of 1:6 and that the relative amplitudes of the intermediate component at cDNA ratios of 1:3 and $3: 1$ should have differed substantially ( 0.26 vs 0.09$)$. None of these predictions is borne out by the results.

The above comparisons indicate that the results can be accounted for if the channels are tetramers and the three distinguishable components correspond to channel assemblies with different stoichiometries of mutant and wild-type subunits. As noted, this analysis rests on the assumption that the relative amplitude of each component accurately reflects the relative abundance of the corresponding channel population. The rise of the currents was substantially faster than the decays, even for the fast component, making it unlikely that we were significantly underestimating the peak amplitudes because the solution exchanges were insufficiently fast. However, because desensitization appears to proceed via closed states (Vyklicky et al., 1991; Raman and Trussell, 1992, 1995), some channels are expected to desensitize without ever opening, and the proportion that do is likely to depend on the rate constant for entry into desensitization. To estimate the extent to which this might confound the analysis, we recorded glutamate-evoked currents in the absence and presence of cyclothiazide, a drug that appears to reduce the rate at which AMPA-type channels enter into desensitization (Partin et al., 1996). Because we found that cyclothiazide caused a modest rundown of the peak currents evoked by prolonged glutamate applications, these experiments were made with a piezoelectric system that allowed us to make glutamate applications of $10 \mathrm{msec}$ or less.

Responses to brief applications of $5 \mathrm{~mm}$ glutamate in the absence and presence of $100 \mu \mathrm{M}$ cyclothiazide are shown in Figure $5 A$. The currents were measured in the same patch from a cell transfected with wild-type GluR $1_{\text {flip }}$. In each of four patches studied, cyclothiazide caused an increase in the peak current through homomeric wild-type channels (mean percentage increase $34 \pm 2 \% ; n=4$ ). This result is predicted for models of the type shown in Figure $6 \mathrm{D}$, where activation and desensitization occur independently from the same closed state. Although the available results are insufficient to define some of the rate constants for the model in Figure $6 D$, at high agonist concentrations the channels spend most of their time in fully liganded states, and this model predicts behavior similar to simpler models proposed previously. Simulations of channel activity evoked by $5 \mathrm{~mm}$ glutamate (using the model in Fig. 6D) showed that the decay of wild-type currents during sustained applications of glutamate could be reproduced with values for channel opening $\left(\beta_{4}\right)$ and channel closing $\left(\alpha_{4}\right)$ of $7000 \mathrm{~s}^{-1}$ and $2550 \mathrm{~s}^{-1}$ and rate constants for entry into and exit from desensitization of $2000 \mathrm{~s}^{-1}\left(\delta_{4}\right)$ and $1 \mathrm{~s}^{-1}\left(\gamma_{4}\right)$. As shown in Figure $5 B$, these values also gave the observed amount of potentiation by cyclothiazide if it was assumed that the effect of cyclothiazide was solely to reduce the rate of entry into desensitization $\left(\delta_{4}=1 \mathrm{~s}^{-1}\right)$ (Partin et al., 1996). Also shown in Figure $5 B$ are simulated currents that give decay time constants that mimic the intermediate and slow components detected in the coexpression experiments. The simulations predict that the peak of the intermediate component more closely approaches that generated by the same number of homomeric mutant channels, because it was necessary to reduce $\delta_{4}$ to slow the decay of this component to the extent observed experimentally. The results of the simulation analysis suggest that the peak amplitudes of the fast and intermediate components underestimate the proportion of channels underlying these phenotypes because significant numbers of channels enter desensitization without opening.

Figure $5 C$ is a plot of the mean relative amplitudes of the fast, intermediate, and slow components of desensitization as a function of the proportion of wild-type cDNA included in the transfection mixture. The smooth curves show the respective probabilities that channel assemblies will contain 0 or 1,2 , and 3 or 4 mutant subunits, assuming that the channels are tetramers. The black curves show the combinatorial predictions. The gray curves show the result of correcting the combinatorial predictions for the likely underestimation of the fast and intermediate components that would occur if some channels desensitize without ever opening. There is good agreement between the combinatorial predictions and the results at each cDNA ratio tested, supporting the 


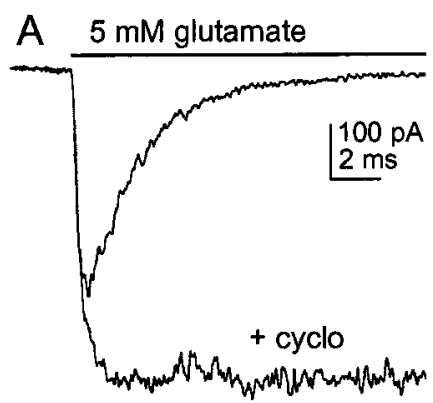

\section{B simulated currents}
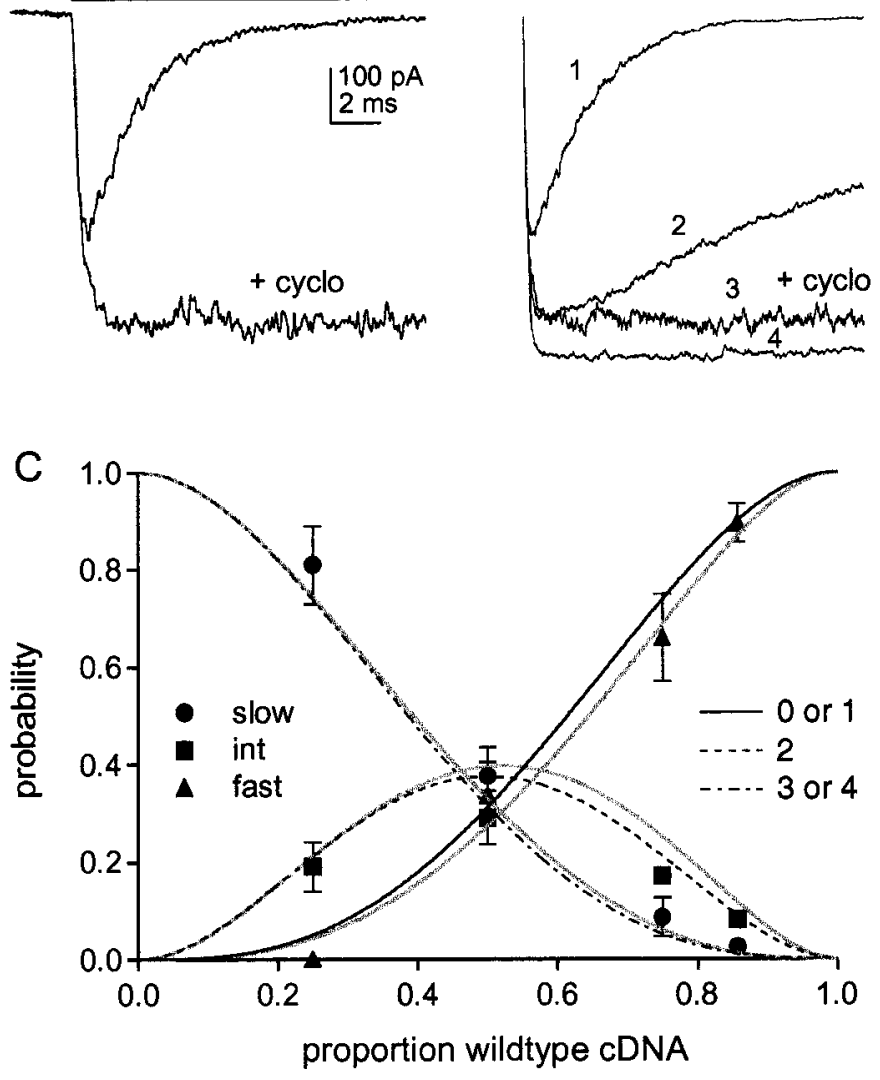

Figure 5. The effect of cyclothiazide and comparison of the results with combinatorial predictions. $A$, Currents evoked by $5 \mathrm{~mm}$ glutamate in a patch from a cell transected with wild-type GluR $1_{\text {flip }}$. Preexposure of the patch to cyclothiazide $(100 \mu \mathrm{M})$ greatly slows desensitization and potentiates the peak current amplitude. $B$, Simulated currents generated with the model shown in Figure $6 D$ in response to a sustained application of 5 mM glutamate. The number of channels was set to 1000 for each simulation. The analysis was performed to estimate the extent to which the peak current amplitude underestimated the number of channels as the result of some channels desensitizing without ever opening. The trace labeled 1 mimics the kinetics of wild-type GluR $1_{\text {flip }}$ homomers. The following values were used for the rate constants: $k_{1}=1 \times 10^{7} \mathrm{M}^{-1} \mathrm{~s}^{-1}, k_{-1}=7000$ $\mathrm{s}^{-1}, \beta_{4}=7000 \mathrm{~s}^{-1}, \alpha_{4}=2550 \mathrm{~s}^{-1}, \delta_{4}=2000 \mathrm{~s}^{-1}, \gamma_{4}=1 \mathrm{~s}^{-1}$. These values reproduced the rise time $(10-90 \%=180 \mu \mathrm{sec})$, desensitization $(\tau=2.2 \mathrm{msec}, 0.25 \%$ plateau current $)$, and deactivation kinetics $\left(\tau_{\text {deact }}=\right.$ $0.7 \mathrm{msec}$ ) (Fig. $6 C$ ) of wild-type channels and gave an $\mathrm{EC}_{50}$ value of $\sim 800$ $\mu \mathrm{M}$. The results were largely insensitive to the values of the other rate constants. The $\beta$ and $\alpha$ values for partially liganded states were set to the corresponding values for the fully liganded state. The conductance of the open states with one, two, and three agonist molecules bound were taken to be $0.25,0.5$, and 0.75 of the value of the fully liganded state. It was assumed that glutamate bound with 100 -fold higher affinity to desensitized channel states $\left(k_{1}=k_{2}, k_{-2}=70 \mathrm{~s}^{-1}\right)$, and the $\delta$ and $\gamma$ values for partially liganded states were set to maintain microscopic reversibility. The trace labeled 3 was obtained with $\delta_{4}=1 \mathrm{~s}^{-1}$ and mimics the effect of cyclothiazide on wild-type channels. The trace labeled 2 mimics the decay of the intermediate component $(\tau=20 \mathrm{msec})$ and was obtained by setting $\alpha_{4}=400 \mathrm{~s}^{-1}$ and $\delta_{4}=1400 \mathrm{~s}^{-1}$. The trace labeled 4 mimics the decay of the slow component $(\tau=100 \mathrm{msec}, 90 \%$ plateau current) and was obtained by setting $\alpha_{4}=200 \mathrm{~s}^{-1}, \delta_{4}=80 \mathrm{~s}^{-1}$, and $\gamma_{4}=15 \mathrm{~s}^{-1}$. These values gave a $\tau_{\text {deact }}$ of $9 \mathrm{msec}$, which is similar to the value measured for homomeric mutant channels (Fig. $6 C$ ). Note that the peak wild-type and intermediate currents (traces 1 and 2) reach only 65 and $88 \%$ of the amplitude of the slow component (trace 4), although the number of channels was set to 1000 for each simulation. $C$, The relative amplitudes of the three components fast $(\mathbf{\Delta})$, intermediate ( $\mathbf{\square})$, and slow $(\boldsymbol{\bullet})$, at the four cDNA ratios tested. Each point is the mean value from measurements made in five to seven patches. The bars indicate the SD, some of which notion that the kinetically distinct components of desensitization arise from channels with different numbers of mutant subunits. Although the cyclothiazide results suggest that the peak fast and intermediate currents underestimate the number of channels by $\sim 35$ and $12 \%$, respectively, this is predicted to have only modest effects on the results. The potential influence of such errors is greatest when all three components are of similar amplitude.

Although we believe that the combinatorial analysis supports the view that AMPA receptors are tetramers, two further observations are worthy of emphasis. First, the time constant of the fast component detected in the coexpression experiments was slower than the corresponding value measured for homomeric wild-type channels $\left(\tau_{\mathrm{f}}=2.24 \mathrm{msec}\right)$, and it became increasingly slower as the relative amount of mutant cDNA was increased $\left(\tau_{\mathrm{f}}=3.04\right.$, 3.52 , and $3.94 \mathrm{msec}$ for mutant/wild-type ratios of 1:6, 1:3, and $1: 1)$. Each of the mean $\tau_{\mathrm{f}}$ values measured in the coexpression experiments was significantly greater than the $\tau_{\mathrm{f}}$ measured for wild-type channels ( $p<0.001$; one-way ANOVA), and the $\tau_{\mathrm{f}}$ values for the $1: 3$ and 1:1 ratios were greater than the value of 3.04 msec obtained at a wild-type/mutant ratio of 1:6 $(p<0.05)$. Second, although there is good agreement in Figure $5 C$ between the predictions and the results, at each cDNA ratio studied the slow component is slightly larger than predicted and the intermediate component is too small. One way to resolve this small, but consistent, discrepancy is to assume that the channels underlying the intermediate component also generate some of the plateau current. However, new structural data suggest an alternative interpretation of the results.

\section{The results are consistent with dimeric subunit assembly}

Armstrong and Gouaux (2000) recently published additional $\mathrm{x}$-ray crystallographic data on the structure of the ligand binding domain of GluR2 and showed that the binding domains crystallize as dimers. Strikingly, two of the residues participating in intersubunit interactions are L483 (L497 for GluR1) and the aspargine residue (N754) that is critical for conferring flop-like sensitivity to cyclothiazide (N750 for GluR1) (Partin et al., 1995). L483 forms a hydrophobic cluster with residues in the flip/flop domain on the adjacent protomer. Because the dimer exhibits twofold symmetry, the L483 residues on adjacent protomers create two spatially distinct connections.

Although Armstrong and Gouaux (2000) were careful to emphasize that their results could reflect a crystal-packing artifact, if AMPA receptor assembly proceeds via the formation of dimers, then all tetrameric assemblies with two mutant subunits are not identical. In one-third of such assemblies the two mutant subunits will be in the same dimer, whereas in the others each dimer will contain one mutant subunit. Figure $6 A$ compares the results with

\section{$\leftarrow$}

were less than half the symbol height. The black curves show the summed probability of homomeric GluR1 $1_{\text {flip }}$ channels and channels with one GluR1 $_{\text {flip }}$ (L497Y) subunit (solid line), the probability that channels will contain two GluR1 $1_{\text {flip }}$ (L497Y) subunits (dotted line), and the summed probability of having homomeric GluR $1_{\text {flip }}$ (L497Y) and channels with one wild-type GluR $1_{\text {flip }}$ subunit (dashed line). The probabilities are plotted as a function of the proportion of wild-type subunits present, assuming that the subunit assemblies are tetramers. The gray curves show the corresponding probabilities after adjusting the results for the fast and intermediate components (for missing channels that never open) using the relative amplitudes of the peak currents predicted from the simulated results in $B$ (fast and intermediate components underestimated by 35 and $12 \%$, respectively, relative to the slow component). 

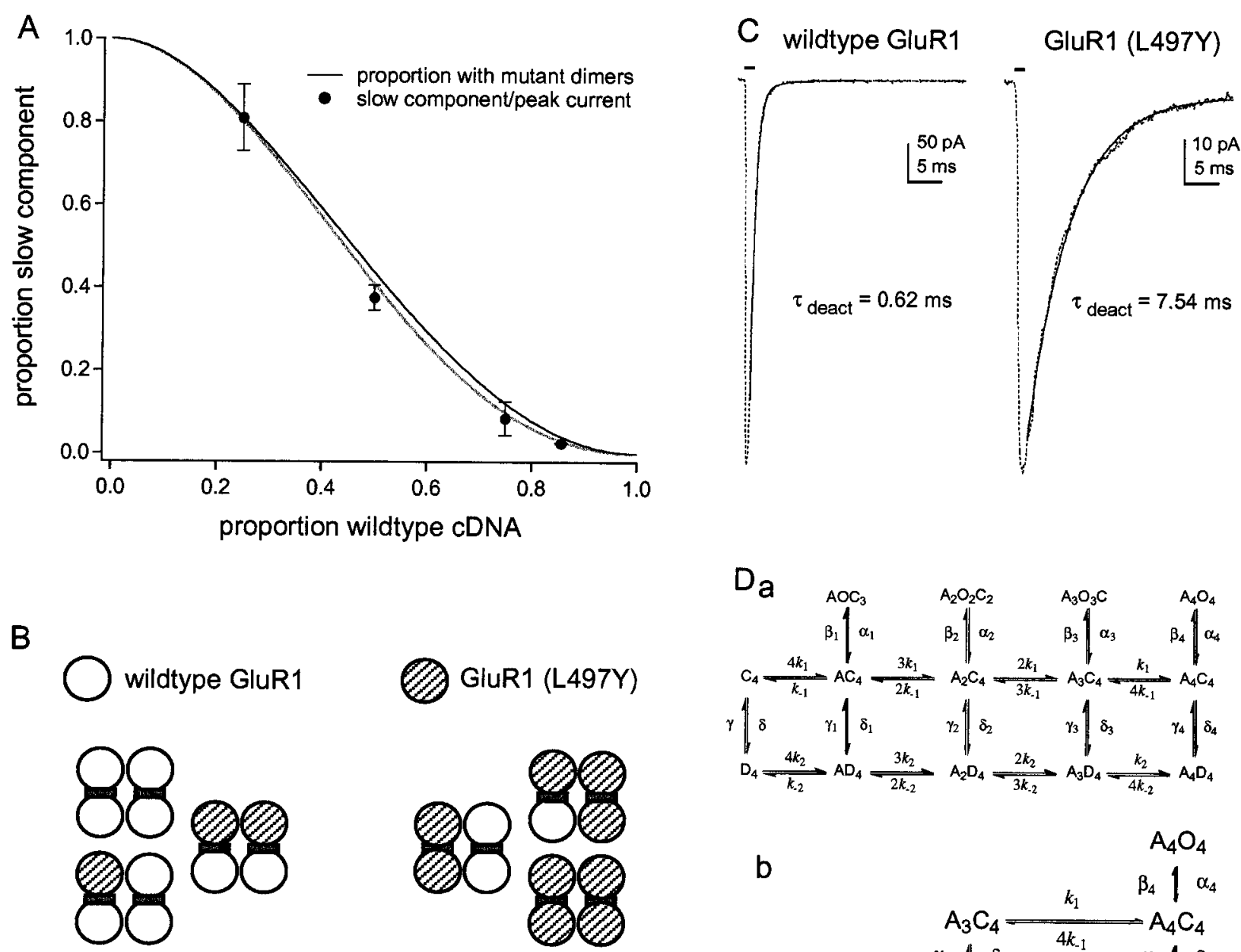

NON-DESENSITIZING

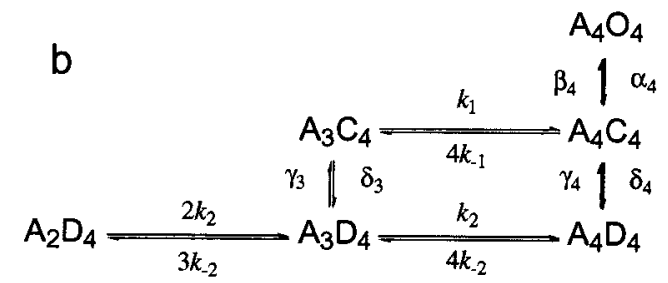

Figure 6. The results are consistent with the conclusion that AMPA receptors are dimers of dimers. $A$, The relative amplitude of the slow component is plotted as a function of the proportion of wild-type cDNA included in the transfection mixture. Each point is the mean value from measurements made in five to seven patches. The bars indicate the SD. The black curve shows the expected proportion of tetramers that contain at least one dimer with two mutant subunits. The gray curve shows the corresponding prediction after correcting for missing channels that never open using the simulated data in Figure $5 B . B$, Diagram illustrating the partitioning of tetrameric assemblies assumed to display wild-type and mutant desensitization for the combinatorial analysis shown in $A$. C, Deactivation kinetics of wild-type GluR1 $1_{\text {flip }}($ left $)$ and homomeric GluR $1_{\text {flip }}(\mathrm{L} 497 \mathrm{Y})$ channels $($ right). The currents were evoked by $1 \mathrm{msec}$ applications (bars) of $5 \mathrm{~mm}$ glutamate. The decays of the currents (dotted traces) were fitted with single exponential functions (solid curves) with the indicated time constants. $D a$, Possible kinetic scheme illustrating some general features of AMPA receptor activation and desensitization suggested by previous work. It is assumed that the channel is a tetramer where each subunit can bind a single agonist molecule $(A)$ and can exist in three distinct conformations: closed $(C)$, open $(O)$, and desensitized $(D)$. The states in the top row are open, those in the middle row are closed, and those in the bottom row are desensitized. For closed and desensitized states, each subunit is constrained to adopt the same conformation. The affinity of agonist binding is higher affinity to desensitized states than to closed states $\left(k_{-2} / k_{2}<k_{-1} / k_{1}\right)$. The top two rows represent one of the models tested by Smith et al. (2000) that incorporates evidence for concentration-dependent substate gating and the presence of as many as four discrete open levels for some native AMPA receptors. The notation of the open states implies that individual subunits gate independently. For simplicity, it is assumed that binding is not cooperative, that binding and unbinding do not occur when the channel is open, that transitions between nonadjacent states do not occur, and that the probability of opening from state $\mathrm{C}_{4}$ is zero. $\mathrm{Db}$, Enlarged view of the scheme showing the portion of the model most relevant to our results.

the combinatorial predictions when it was assumed that all tetramers containing at least one mutant dimer (both subunits with the L497Y mutation) were non-desensitizing, whereas the extent to which other heteromeric channels desensitized was indistinguishable from wild-type homomers (Fig. 6B). As can be seen, the relative amplitude of the slow component agrees well with the proportion of channels that contains at least one mutant dimer at each cDNA ratio tested (Fig. 6A). Correction for missing channels that never open (gray curve) produces even better agreement. Thus the size of the slow component can be accounted for if it is assumed that the non-desensitizing phenotype of GluR $1_{\text {flip }}$
(L497Y) is dominant, but that this dominance requires that at least one dimer in the tetramer contains two mutant subunits.

\section{Slow desensitization versus slow deactivation}

Although the "dominant dimer" interpretation accounts for the dependence of the size of the slow component on the relative abundance of wild-type and mutant subunits, it does not explain the intermediate component of decay. However, as emphasized by Partin et al. (1996), the decay of AMPA receptor currents in the continuous presence of agonist is determined by the rate of both deactivation and desensitization. Previous work on wild- 
type GluR1 homomers has given deactivation time constants of $\sim 0.8 \mathrm{msec}$ (Partin et al., 1996). In contrast, the deactivation rate of homomeric GluR $1_{\text {flip }}$ (L497Y) channels is much slower. Figure $6 C$ illustrates the decay of currents through wild-type and mutant homomers after termination of a $1 \mathrm{msec}$ application of $5 \mathrm{~mm}$ glutamate. The decays have been fitted with single exponential functions that give time constants that differ by more than an order of magnitude. On average, the mean time constant of deactivation was $0.68 \pm 0.07 \mathrm{msec}$ for wild-type channels and $8.9 \pm 0.9 \mathrm{msec}$ for channels carrying the L497Y mutation $(n=5$ and 4 patches, respectively). Thus, in addition to altering desensitization, the L497Y mutation also produces a $\sim 13$-fold slowing of deactivation.

Two commonly agreed upon features in previously published models of AMPA receptor kinetics are that desensitization occurs exclusively from closed states and that the rate constant for channel opening is greater than the rate constant for entry into desensitization (Vyklicky et al., 1991; Raman and Trussell, 1992, 1995; Partin et al., 1996). Figure $6 D$ illustrates a kinetic scheme that incorporates these features and extends them to account for concentration-dependent substate gating and the accumulating evidence that AMPA receptors are tetramers (Mano and Teichberg, 1998; Rosenmund et al., 1998; Smith and Howe, 2000). In this scheme, the observed decay during a sustained application of a saturating concentration of agonist will be determined not just by the rate constant for entry into desensitization $\left(\delta_{4}\right)$ but also by the length of individual openings $\left(1 / \alpha_{4}\right)$ and the average number of times the channel opens before desensitization occurs. Comparisons of the time constants of deactivation and desensitization for various AMPA-type channels suggest that, on average, the fully liganded receptor will open and close multiple times before it desensitizes (Partin et al., 1996). Depending on the relative values of $k_{-1}, \beta_{4}$, and $\delta_{4}$, it is also possible that the apparent slowing of deactivation might reflect a decrease in the rate of entry into desensitization $\left(\delta_{4}\right)$.

To gain some insight into the effect of the L497Y on the various microscopic rate constants that could influence operational measurements of the time constants of deactivation and desensitization, we performed channel simulations and systematically altered the rate constants for transitions into and out of fully liganded channel states. The analysis indicated that the slowing of deactivation could be largely accounted for if it was assumed that it arose from alterations in $\alpha_{4}$, the rate constant for channel closure. In contrast, independent alterations in $k_{-1}, \beta_{4}$, and $\delta_{4}$ failed to reproduce the results. For example, slowing $\gamma_{4}$ to the extent required to reproduce the mutant desensitization phenotype resulted in virtually no change in deactivation, and increasing $\beta_{4}$ to an extent sufficient to slow both deactivation and desensitization produced activation time courses that were unrealistic. Alterations in $k_{-1}$ that markedly slowed desensitization gave biphasic deactivation decays caused by the interspersion of substate openings between sequential unbinding steps. In contrast, reducing $\alpha_{4}$ by a factor of 13 not only accounted for the slowing of deactivation in the L497Y mutant channels, but it slowed the decay of the current during a sustained application of glutamate to a similar extent. We have not measured the deactivation rate for the intermediate component of decay. However, if the slowing of deactivation depends on the stoichiometry of wild-type and mutant subunits, then this would largely explain the appearance of the intermediate component. If channels containing a single mutant subunit deactivate somewhat more slowly than wild-type channels, the apparent slowing of the fast component of decay could reflect the increasing (but unresolved) contribution of this slower component as the relative abundance of mutant subunit was increased. To test this latter idea, we generated decays that contained four components and then fit them with only three exponentials. We found that the slowing of the fast time constant of desensitization could be reproduced if it was assumed that the currents through channels containing one mutant subunit decay with a time constant of $\sim 4$ msec (twofold reduction in $\alpha_{4}$ ).

\section{Intermediate channels recover faster from desensitization}

It has been proposed that the entry into, and recovery from, desensitization may proceed via different paths (Patneau and Mayer, 1991; Jonas, 1993; Raman and Trussell, 1995). To test whether the inclusion of the mutant subunit in receptor assemblies also altered the rate of recovery from desensitization, we compared the speed of recovery of the intermediate and fast components. For these experiments, we used patches from cells transfected with mutant and wild-type cDNAs at a ratio of 1:3, which showed substantial fast and intermediate components in their decays. Patches were conditioned with an application of glutamate $(0.5 \mathrm{~mm}$ for $100 \mathrm{msec})$, and then a second application was delivered at varying times after the conditioning pulse.

A typical example of the results obtained in these experiments is shown in Figure $7 A$. The decays of the currents evoked by the conditioning and test pulses were each fitted with three exponential components. The amplitudes of the fast and intermediate components in the decay of the current evoked by the test pulse were then expressed as a proportion of the amplitude of the corresponding component in the decay of the current evoked by the conditioning pulse. Figure $7 B$ shows a plot of the relative amplitudes of the fast and intermediate components as a function of recovery time, where each point is the mean value obtained from five patches. Single exponential fits to each set of results gave recovery time constants $\left(\tau_{\text {rec }}\right)$ of $154 \mathrm{msec}$ for the fast current and $51 \mathrm{msec}$ for the intermediate current. The apparent offset (see Materials and Methods) was similar for both sets of data (19 and $21 \mathrm{msec}$ ). Because the intermediate component of decay appears to arise from channels that contain two mutant subunits, the data from the two-pulse experiments suggest that these channels also recover from desensitization faster than wild-type channels.

At high agonist concentrations, recovery from desensitization could occur directly to non-desensitized states or it might proceed initially via unbinding transitions, with exit from desensitization only occurring after agonist has dissociated from one or more subunits. Thus the somewhat faster recovery of the intermediate component could either reflect modest destabilization of desensitized channel states (increase in $\gamma_{4}$ ) (Fig. 6D) or a decreased affinity of glutamate binding to these states (increase in $k_{-2}$ ). To distinguish between these two possibilities, we determined the apparent affinity of glutamate for desensitized channel states. As reported by others (Trussell and Fischbach, 1989; Colquhoun et al., 1992; Raman and Trussell, 1992), preincubation of wild-type channels with low concentrations of glutamate (which do not cause detectable channel activation) produces significant desensitization, as assessed by depression of the currents evoked by subsequent glutamate applications. The data in Figure $7 C$ gave an $\mathrm{IC}_{50}$ value of $0.89 \mu \mathrm{M}$ for glutamate depression of glutamateevoked currents through wild-type GluR1flip channels $(n=4$ patches). The corresponding value measured for the intermediate component of decay was $4.3 \mu \mathrm{M}(n=5$ patches $)$. An example of 
A
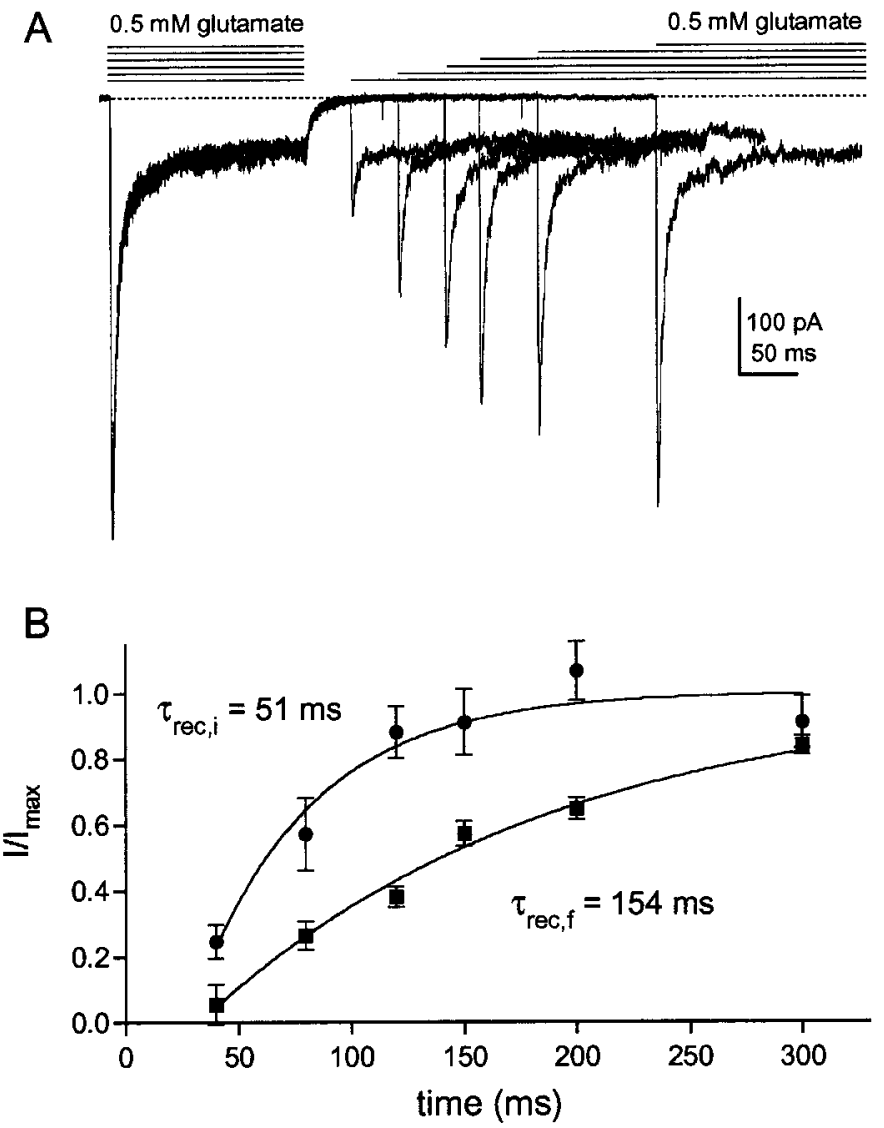

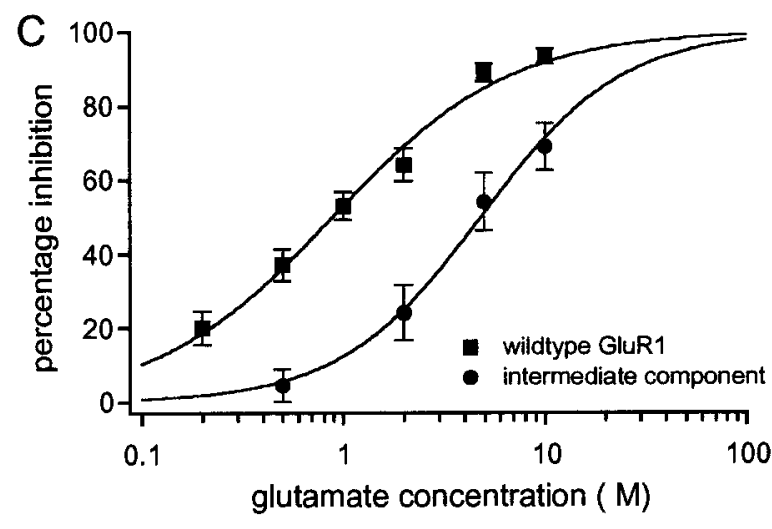

D

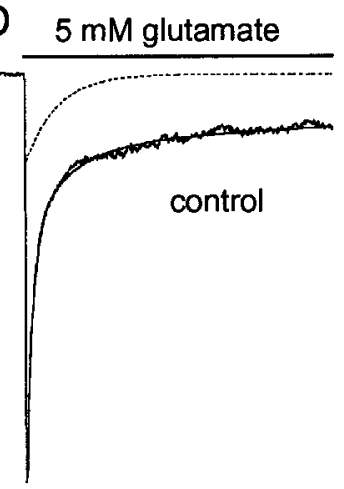

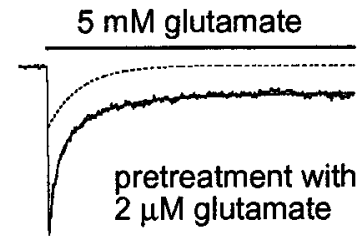

$25 \mathrm{pA}$

$50 \mathrm{~ms}$

Figure 7. The intermediate component recovers faster from desensitization and shows reduced apparent affinity for glutamate. $A$, Six consecutive sweeps obtained in a patch from a cell that was cotransfected with GluR1 $1_{\text {flip }}$ (L497Y) and GluR $1_{\text {flip }}$ CDNAs at a ratio of 1:3. Glutamate $(0.5$ mM) was applied for $100 \mathrm{msec}$, and then a second application was made at a varying interval $(40,80,120,150,200,300 \mathrm{msec})$. The decays of the currents evoked by the second application of each pair were fitted with three exponential components, and their amplitudes were expressed as a proportion of the corresponding component in the decay of the current evoked by the preceding conditioning pulse. $B$, Graph showing the recovery from desensitization of the fast ( $\square$ ) and the intermediate () components. Each point is the mean from five different patches (bars indicate SD). Each set of results was fitted with a single exponential function. The fits did not extrapolate to zero time. This apparent offset was estimated from the fit and was subtracted from all recovery times. $C$, Percentage inhibition of peak currents through wild-type $\mathrm{GluR}_{\text {flip }}$ channels $(\boldsymbol{\square})$ are plotted as a function of glutamate concentration and compared with the corresponding results for the intermediate component detected in coexpression experiments $(\bullet)$. Each point is the mean value obtained from five patches (bars indicate SD). Each data set was fitted with a Hill-type equation with the maximal inhibition constrained to $100 \%$. In the coexpression experiments, glutamate concentrations above $10 \mu \mathrm{M}$ produced significant channel activation. The fits gave $\mathrm{IC}_{50}$ values of $0.89 \mu \mathrm{M}$ for the wild-type channels and $4.30 \mu \mathrm{M}$ for the channels underlying the intermediate component of decay. $D$, Currents in a patch from a cell transfected with GluR1 $1_{\text {flip }}(\mathrm{L} 497 \mathrm{Y})$ and GluR1 $1_{\text {flip }}$ at a cDNA ratio of 1:3. The records are responses to 5 mm glutamate before and during preincubation with $2 \mu \mathrm{M}$ glutamate. The three-exponential fits to the decays (smooth solid lines) are superposed on the currents, and the dotted lines show the intermediate component obtained from each fit. Note that preincubation with glutamate reduces the amplitude of the fast component more than it does the amplitude of the intermediate component (71 and $30 \%$ inhibition, respectively).

the preincubation results obtained in one of the patches is shown in Figure $7 D$. Preincubation of the patch with $2 \mu \mathrm{M}$ glutamate resulted in a substantial reduction in the fast component of decay with much less effect on the amplitude of the intermediate component. The results support the conclusion that the desensitized states of channels underlying the intermediate component exhibit four- to fivefold lower affinity for glutamate (compared with wild-type channels), and they suggest that this reduced affinity is why the channels recover faster from desensitization.

\section{The expression of GluR1 $1_{\text {flip }}$ (L497Y) in neurons also gives an intermediate phenotype}

From studies in oocytes, Thalhammer et al. (1999) concluded that the L497Y mutation is dominant, the presence of a single mutant subunit being sufficient to render various types of heteromeric AMPA receptors non-desensitizing. This conclusion appears at odds with our results. Therefore, to determine whether the inter- mediate desensitization behavior that we observed after coexpression of GluR $1_{\text {flip }}$ with GluR $1_{\text {flip }}$ (L497Y) was unique to channels containing exclusively flip versions of GluR1, we expressed the GluR $1_{\text {flip }}$ (L497Y) subunit in cerebellar granule cell neurons. Primary cultures of rat cerebellum were maintained in vitro for 6-8 d and transiently transfected with GluR $1_{\text {flip }}$ (L497Y). Granule cells expressing the mutant subunit (identified by coexpressing the GFP) represented $1-2 \%$ of the total number of cells per coverslip. The decay kinetics of responses to glutamate (2 mM) were determined in patches pulled from transfected granule cells and compared with the kinetics of responses obtained in patches from untransfected cells on the same coverslip.

Typical currents evoked by the application of $2 \mathrm{~mm}$ glutamate in patches from control and transfected cells are shown in Figure 8. As illustrated in Figure $8 A$, currents in patches pulled from control cells showed rapid and virtually complete desensitization. 
Aa

$2 \mathrm{mM}$ glutamate

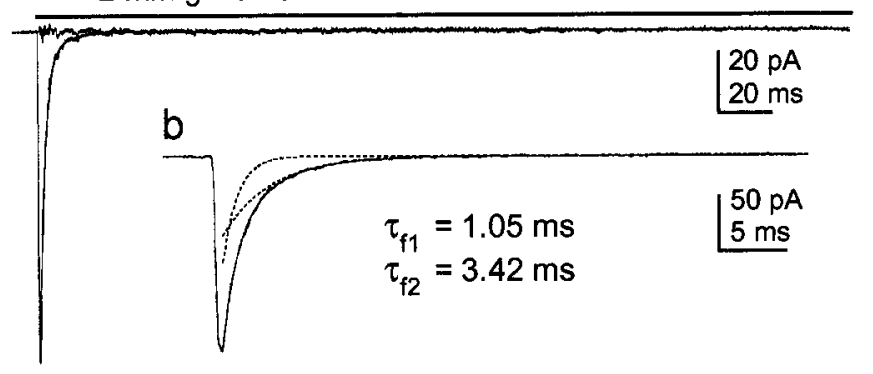

\section{Ba 2 mM glutamate}

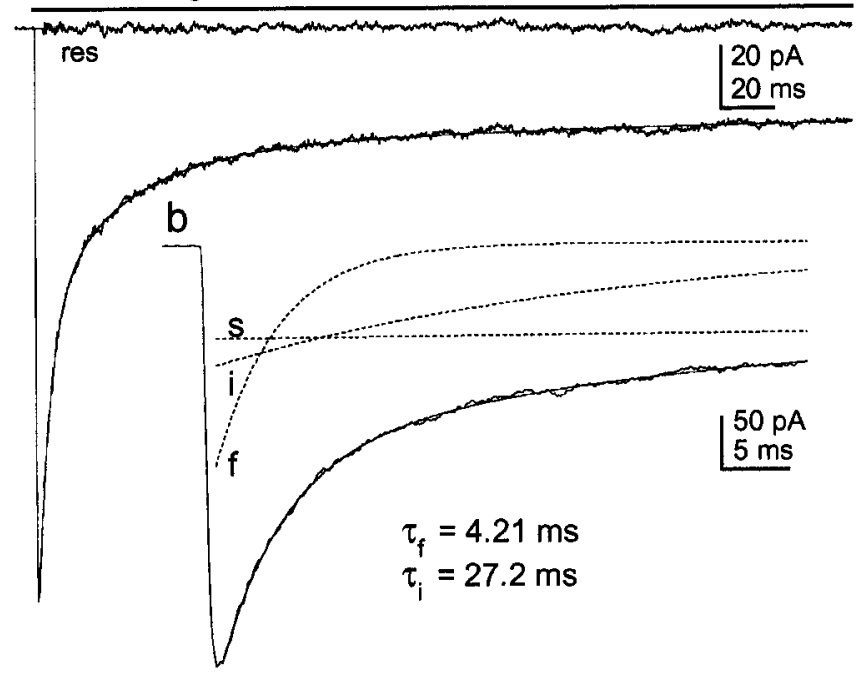

Figure 8. The assembly of GluR $1_{\text {flip }}$ (L497Y) with native subunits also generates intermediate desensitization. $A a$, Current activated by glutamate $(2 \mathrm{mM})$ in an outside-out patch from a control granule cell maintained in culture for $7 \mathrm{~d}$. The decay of the current was fitted with a function consisting of two exponentials (smooth solid line). Ab, Same current on an expanded time scale with the individual components superposed (dotted lines). The time constants of the two components are given on the figure. $B a$, Current activated by $2 \mathrm{~mm}$ glutamate in a patch from a granule cell that was maintained in vitro for $7 \mathrm{~d}$ and transiently transfected $24 \mathrm{hr}$ before with GluR $1_{\text {flip }}$ (L497Y). The decay of the current was fitted with a function consisting of three exponentials and a sustained current (smooth solid line). Bb, The current, the fit, and the three individual exponential components are shown on an enlarged time scale. The time constants for the fast and intermediate components are given. Note that the fast component is slower than the fast component in the control neuron.

The decays of these currents contained two exponential components. The fits to results obtained in eight control patches gave mean time constants for these components of $1.05 \pm 0.09$ and $3.82 \pm 0.15 \mathrm{msec}$. On average, the amplitude of the fast and slower components represented $66 \pm 1.4$ and $34 \pm 1.8 \%$ of the total peak current. Granule cells express predominantly the GluR2 and GluR4 subunits (Monyer et al., 1991). The time constant of the fast component $(\tau \approx 1 \mathrm{msec})$ agrees well with the corresponding value for channels containing the GluR $4_{\text {flop }}$ subunit (with or without GluR2), whereas the slower component $(\tau \approx$ $4 \mathrm{msec}$ ) is similar to the desensitization rate of channels that contain GluR4 $4_{\text {flip }}$ (with or without the flop version of GluR2) (Mosbacher et al., 1994). Thus the fast and slow components likely arise from channels containing the flop and flip versions of
GluR4, respectively. None of the patches gave plateau currents that were $>1 \%$ of the total peak current.

As shown in Figure $8 B$, the currents evoked by glutamate in patches pulled from neurons expressing the GluR1 $1_{\text {flip }}$ (L497Y) subunit displayed a large sustained component that was clearly not present in the patches pulled from control granule cells. The decays of these currents contained three exponential components with time constants of $3.61 \pm 0.24,26.5 \pm 2.4$, and $180 \pm 20 \mathrm{msec}$ ( $n=5$ patches). In all the patches, both the slow component (including the plateau current) and the intermediate component represented $>20 \%$ of the total peak current. The similarity of the intermediate component of decay in transfected neurons to the intermediate component seen in the cotransfection experiments strongly supports the conclusion that it arises from heteromeric assembly of GluR1 $1_{\text {flip }}$ (L497Y) with native AMPA receptor subunits. Indeed, the decays of the responses in transfected neurons also showed a slowing of the fast component of decay, and they were strikingly similar to those observed in HEK 293 cells (compare Figs. 3 and 8). This similarity suggests that our observations with recombinant channels are not restricted to GluR1 homomeric channels, nor are they likely to be unique to channels composed solely of flip splice variants.

\section{DISCUSSION}

The coexpression of GluR $1_{\text {flip }}$ with the non-desensitizing mutant subunit GluR1 $1_{\text {flip }}$ (L497Y) produced channels that appear to desensitize at three kinetically distinct rates, one of which is intermediate between that of wild-type and mutant homomeric channels. The relative amplitudes of the three components were described well by simple combinatorial predictions if the channels were assumed to be tetramers. In contrast, there was no partitioning of the combinatorial probabilities that accounted well for the results if the channels were assumed to be pentamers.

\section{Allosteric models of desensitization and subunit dominance}

Allosteric models of the Monod-Wyman-Changeaux (MWC) type (Monod et al., 1965) have been advanced to explain the behavior of various ion channels. Here allosteric implies, among other things, that each subunit in the oligomer can exist in at least two distinct conformations, all subunits are constrained to adopt the same conformation, and the ligand binds to one subunit conformation with higher affinity than to the others (for review, see Colquhoun, 1998).

It has been noted that the desensitization of AMPA receptors shows features reminiscent of such allosteric models. First, concentrations of glutamate insufficient to activate AMPA receptors can produce nearly complete AMPA receptor desensitization (Trussell and Fischbach, 1989; Patneau and Mayer, 1991; Colquhoun et al., 1992; Raman and Trussell, 1992), supporting the idea that glutamate binds with highest affinity to the desensitized form of the receptor. Second, evidence supporting a concerted conformational change comes from results showing that some desensitization phenotypes are dominant in heteromeric assemblies. For example, the GluR $4_{\text {flop }}$ subunit determines the rate of desensitization in heteromeric channels (Mosbacher et al., 1994), and the presence of a single flip-type subunit appears sufficient to confer flip-type sensitivity to cyclothiazide (Partin et al., 1994, 1995).

One interpretation of our results is that the rate and extent of desensitization titrates with the number of non-desensitizing subunits. Although this interpretation conflicts with the notion of 
subunit dominance, graded stoichiometric changes do not necessarily argue against allosteric mechanisms. If the conformational change corresponding to desensitization is concerted, then it might become less likely as the number of "reluctant" subunits in the oligomer is increased and thereby result in a progressive slowing of the rate at which the population of channels desensitizes.

The results are described just as well, however, by the "dominant dimer" analysis presented in Figure 6. This interpretation is consistent with recent structural data (Armstrong and Gouaux, 2000) and also better reconciles our results with the evidence described above for subunit dominance. If this view is correct, then the decay of the intermediate component must be largely the result of the slowing of deactivation. Our simulation results suggest that the decay of the intermediate component can be accounted for with only modest changes in the rate constant for entry into desensitization ( $\delta_{4}$ decreased less than twofold) if it is assumed that channels with one mutant subunit in each dimer deactivate sixfold more slowly than wild-type channels. Although this result somewhat supports the dominant dimer hypothesis, at present our results do not allow us to exclude other interpretations.

The crystallographic data on the ligand binding domain of GluR2 demonstrate that the dimers show twofold symmetry (Armstrong and Gouaux, 2000). If AMPA receptors display similar symmetry, then they would be expected to form tetramers in which the protomers associate exclusively via isologous and symmetrical interactions. As pointed out by Monod et al. (1965) in their seminal paper on allosteric transitions, in such symmetrical oligomers each protomer is subject to the same quaternary constraints, and therefore all protomers are likely to adopt the same conformation.

\section{The L497Y mutation appears to destabilize desensitized states}

Although the slowing of deactivation accounts substantially for the intermediate component of decay, it cannot account for the substantial plateau current generated by channels containing three or four mutant subunits. This greatly reduced steady-state desensitization must reflect alterations in the rate constants for entry into, or recovery from, desensitized channel states. The L497Y mutation in GluR1 does not remove desensitization completely. Currents through homomeric mutant channels decayed with a time constant of $\sim 100 \mathrm{msec}$ to $85-90 \%$ of their peak amplitude (Fig. 1Ba). We found that reproducing this phenotype in channel simulations required substantial changes in the values of both $\delta_{4}$ and $\gamma_{4}$ ( $\sim 15$-fold). Indeed this analysis suggests that it is the increased rate of exit from desensitization that is largely responsible for the "non-desensitizing" phenotype, because the channels still enter desensitization frequently, but they return from desensitization nearly as often.

Armstrong and Gouaux (2000) suggested that the leucine-totyrosine mutation studied here might stabilize interactions between adjacent protomers [see also Partin (2001)]. In each protomer, the two globular domains surrounding the glutamate receptor binding pocket are thought to close around the ligand in "venus flytrap" models of ligand receptor binding (for review, see Dingledine et al., 1999; Howe, 1999). Interestingly, the amount of domain closure is ligand dependent, being smallest for antagonists, intermediate for the partial and incompletely desensitizing agonist kainate, and greatest for full and strongly desensitizing agonists such as glutamate and AMPA (Armstrong et al., 1998;
Armstrong and Gouaux, 2000). By strengthening intersubunit interactions, the L497Y mutation might restrict domain closure within individual subunits. If this is true, and the L497Y mutation speeds escape from desensitized states, then domain closure may not initiate desensitization but rather stabilize the resulting nonconducting conformations.

\section{Effects on apparent affinity}

For cyclic models of the type shown in Figure $6 D$, it is expected that altering desensitization will alter binding affinity (and vice versa). This is so because to maintain microscopic reversibility the $\delta / \gamma$ ratios for adjacent paths must differ by the difference in the equilibrium dissociation constants for binding to closed versus desensitized states [for example: $\left(\delta_{4} / \gamma_{4}\right) /\left(\delta_{3} / \gamma_{3}\right)=\left(k_{-1} / k_{1}\right) /\left(k_{-2} /\right.$ $k_{2}$ ) (Fig. $\left.6 D\right)$ ]. In line with these expectations, cyclothiazide both removes desensitization and markedly increases the apparent affinity of agonists for non-desensitized closed states (Patneau et al., 1993; Yamada and Tang, 1993; Kessler et al., 1996). Previous studies also suggest that the extent of desensitization (as assessed by the ratio of the peak to plateau current) increases with agonist concentration (Geoffroy et al., 1991; Vyklicky et al., 1991).

The $\mathrm{EC}_{50}$ value that we determined for glutamate activation of homomeric GluR1 $1_{\text {flip }}$ (L497Y) channels ( $28 \mu \mathrm{M}$; data not shown) is $\sim 30$-fold smaller than the corresponding value for wild-type GluR1 channels when desensitization is intact (Partin et al., 1996; A. Robert and J. R. Howe, unpublished observations). Our results also suggest that the inclusion of L497Y subunits in heteromeric assemblies reduces the affinity with which glutamate binds to desensitized channel states (Fig. 7C). Thus the L497Y mutation both reduces desensitization and results in changes in relative affinity that are qualitatively those expected for allosteric schemes. How tightly alterations in desensitization are necessarily linked to alterations in agonist binding affinity, and how our results relate to the concentration dependence of desensitization, are questions that require further investigation.

The intermediate component of decay showed hastened recovery from desensitization, and the apparent affinity of glutamate for desensitized channel states was reduced to a similar extent as recovery from these states was speeded. These results suggest that unbinding steps precede exit from desensitization for the channels underlying the fast and intermediate components. Studies on native channels support a similar conclusion (Patneau and Mayer, 1991).

\section{Allosteric versus subunit independent gating}

Our results support the idea that AMPA receptor desensitization proceeds via an allosteric mechanism, and they provide functional evidence consistent with dimeric receptor assembly. AMPA receptors also show concentration-dependent substate gating (Rosenmund et al., 1998; Smith and Howe, 2000), a property shared by cyclic nucleotide-gated $(\mathrm{CNG})$ channels (Ruiz and Karpen, 1997). Interestingly, it appears that CNG channels also behave as dimers of dimers and obey allosteric gating mechanisms, but for CNG channels it is activation, not desensitization, that is described by modified MWC schemes (Liu et al., 1998; but see Ruiz and Karpen, 1999).

Individual AMPA receptors show as many as four conductance levels, the frequency of occurrence of which depends on the number of subunits occupied by agonist (Smith and Howe, 2000; Smith et al., 2000). One interpretation of these results, and the staircase behavior described by Rosenmund et al. (1998), is that individual subunits gate independently of the state of the other 
subunits in the tetramer. Thus activation may differ fundamentally from desensitization, the latter being a process that appears to require a concerted conformational change involving all four subunits (or both dimers). That activation and desensitization are distinct and separable processes is implicit in previous kinetic schemes (Raman and Trussell, 1995; Partin et al., 1996) and was also a main conclusion of the mutagenesis work of Stern-Bach et al. (1998).

\section{REFERENCES}

Armstrong N, Gouaux E (2000) Mechanisms for activation and antagonism of an AMPA-sensitive glutamate receptor: crystal structures of the GluR2 ligand binding core. Neuron 28:165-181.

Armstrong N, Sun Y, Chen GQ, Gouaux E (1998) Structure of a glutamate-receptor ligand-binding core in complex with kainate. Nature 395:913-917.

Banke TG, Bowie D, Lee H, Huganir RL, Schousboe A, Traynelis SF (2000) Control of GluR1 AMPA receptor function by cAMPdependent protein kinase. J Neurosci 20:89-102.

Colquhoun D (1998) Binding, gating, affinity and efficacy: the interpretation of structure-activity relationships for agonists and of the effects of mutating receptors. Br J Pharmacol 125:924-947.

Colquhoun D, Jonas P, Sakmann B (1992) Action of brief pulses of glutamate on AMPA/kainate receptors in patches from different neurones of rat hippocampal slices. J Physiol (Lond) 458:261-287.

Derkach V, Barria A, Soderling TR (1999) Ca2+/calmodulin-kinase II enhances channel conductance of alpha-amino-3-hydroxy-5-methyl-4isoxazolepropionate type glutamate receptors. Proc Natl Acad Sci USA 96:3269-3274.

Dingledine R, Borges K, Bowie D, Traynelis SF (1999) The glutamate receptor ion channels. Pharmacol Rev 51:7-61.

Geoffroy M, Lambolez B, Audinat E, Hamon B, Crepel F, Rossier J, Kado RT (1991) Reduction of desensitization of a glutamate ionotropic receptor by antagonists. Mol Pharmacol 39:587-591.

Horn R (1987) Statistical methods for model discrimination. Application to gating kinetics and permeation of the acetylcholine receptor. Biophys J 51:255-263.

Howe JR (1996) Ion channels formed from the kainate-type subunits GluR6 and KA2 have very small, but different, unitary conductances. J Neurophysiol 76:510-519.

Howe JR (1999) How glutamate receptors are built. The Neuroscientist $5: 311-323$.

Jonas P (1993) AMPA-type glutamate receptors-nonselective cation channels mediating fast excitatory transmission in the CNS. EXS 66:61-76.

Jonas P, Spruston N (1994) Mechanisms shaping glutamate-mediated excitatory postsynaptic currents in the CNS. Curr Opin Neurobiol 4:366-372.

Jones MV, Westbrook GL (1996) The impact of receptor desensitization on fast synaptic transmission. Trends Neurosci 19:96-101.

Kessler M, Arai A, Quan A, Lynch G (1996) Effect of cyclothiozide on binding properties of AMPA-type glutamate receptors: lack of competition between cyclothiazide and GYKI 52466. Mol Pharmacol 49:123-131.

Liu DT, Tibbs GR, Paoletti P, Siegelbaum SA (1998) Constraining ligand-binding site stoichiometry suggests that a cyclic nucleotide-gated channel is composed of two functional dimers. Neuron 21:235-248.

Lomeli H, Mosbacher J, Melcher T, Hoger T, Geiger JR, Kuner T, Monyer H, Higuchi M, Bach A, Seeburg PH (1994) Control of kinetic properties of AMPA receptor channels by nuclear RNA editing. Science 266:1709-1713.

Mano I, Teichberg VI (1998) A tetrameric subunit stoichiometry for a glutamate receptor-channel complex. NeuroReport 9:327-331.

Mano I, Lamed Y, Teichberg VI (1996) A venus flytrap mechanism for activation and desensitization of alpha-amino-3-hydroxy-5-methyl-4isoxazole propionic acid receptors. J Biol Chem 271:15299-15302.

Marshall J, Molloy R, Moss GWJ, Howe JR, Hughes TE (1995) The jellyfish green fluorescent protein: a new tool for studying ion channel expression. Neuron 14:211-215.
Monod J, Wyman J, Changeux JP (1965) On the nature of allosteric transitions: a plausible model. J Mol Biol 12:88-118.

Monyer H, Seeburg PH, Wisden W (1991) Glutamate-operated channels: developmentally early and mature forms arise by alternative splicing. Neuron 6:799-810.

Mosbacher J, Schoepfer R, Monyer H, Burnashev N, Seeburg PH, Ruppersberg JP (1994) A molecular determinant for submillisecond desensitization in glutamate receptors. Science 266:1059-1062.

Otis T, Zhang S, Trussell LO (1996) Direct measurement of AMPA receptor desensitization induced by glutamatergic synaptic transmission. J Neurosci 16:7496-7504.

Partin KM (2001) Domain interactions regulating AMPA receptor desensitization. J Neurosci 21:1939-1948.

Partin KM, Patneau DK, Winters CA, Mayer ML, Buonanno A (1993) Selective modulation of desensitization at AMPA versus kainate receptors by cyclothiazide and concanavalin A. Neuron 11:1069-1082.

Partin KM, Patneau DK, Mayer ML (1994) Cyclothiazide differentially modulates desensitization of alpha-amino-3-hydroxy-5-methyl-4isoxazolepropionic acid receptor splice variants. Mol Pharmacol 46:129-138.

Partin KM, Bowie D, Mayer ML (1995) Structural determinants of allosteric regulation in alternatively spliced AMPA receptors. Neuron 14:833-843.

Partin KM, Fleck MW, Mayer ML (1996) AMPA receptor flip/flop mutants affecting deactivation, desensitization, and modulation by cyclothiazide, aniracetam, and thiocyanate. J Neurosci 16:6634-6647.

Patneau DK, Mayer ML (1991) Kinetic analysis of interactions between kainate and AMPA: evidence for activation of a single type of receptor in mouse hippocampal neurons. Neuron 6:785-798.

Patneau DK, Vyklicky L, Mayer ML (1993) Hippocampal neurons exhibit cyclothiazide-sensitive rapidly desensitizing responses to kainate. J Neurosci 13:3496-3509.

Qin F, Auerbach A, Sachs F (1996) Estimating single-channel kinetic parameters from idealized patch clamp data containing missed events. Biophys J 70:264-280.

Qin F, Auerbach A, Sachs F (1997) Maximum likelihood estimation of aggregated Markov processes. Proc R Soc Lond B Biol Sci 264:375-383.

Raman IM, Trussell LO (1992) The kinetics of the response to glutamate and kainate in neurons of the avian cochlear nucleus. Neuron 9:173-186.

Raman IM, Trussell LO (1995) The mechanism of alpha-amino-3hydroxy-5-methyl-4-isoxazolepropionate receptor desensitization after removal of glutamate. Biophys J 68:137-146.

Rosenmund C, Stern-Bach Y, Stevens CF (1998) The tetrameric structure of a glutamate receptor channel. Science 280:1596-1599.

Ruiz M, Karpen JW (1997) Single cyclic nucleotide-gated channels locked in different ligand-bound states. Nature 389:389-392.

Ruiz M, Karpen JW (1999) Opening mechanism of a cyclic nucleotidegated channel based on analysis of single channels locked in each liganded state. J Gen Physiol 113:873-895.

Smith TC, Howe JR (2000) Concentration-dependent substate behavior of native AMPA receptors. Nat Neurosci 3:992-997.

Smith TC, Wang LY, Howe JR (2000) Heterogeneous conductance levels of native AMPA receptors. J Neurosci 20:2073-2085.

Sommer B, Keinanen K, Verdoorn TA, Wisden W, Burnashev N, Herb A, Kohler M, Takagi T, Sakmann B, Seeburg PH (1990) Flip and flop: a cell-specific functional switch in glutamate-operated channels of the CNS. Science 249:1580-1585.

Stern-Bach Y, Russo S, Neuman M, Rosenmund C (1998) A point mutation in the glutamate binding site blocks desensitization of AMPA receptors. Neuron 21:907-918.

Thalhammer A, Morth T, Strutz N, Hollmann M (1999) A desensitization-inhibiting mutation in the glutamate binding site of rat alpha-amino-3-hydroxy-5-methyl-4-isoxazole propionic acid receptor subunits is dominant in heteromultimeric complexes. Neurosci Lett 277:161-164

Trussell LO, Fischbach GD (1989) Glutamate desensitization and its role in synaptic transmission. Neuron 3:209-218.

Vyklicky Jr L, Patneau DK, Mayer ML (1991) Modulation of excitatory synaptic transmission by drugs that reduce desensitization at AMPA/ kainate receptors. Neuron 7:971-984.

Yamada KA, Tang C-M (1993) Benzothiazides inhibit rapid glutamate receptor desensitization and enhance glutamatergic synaptic currents. J Neurosci 13:3904-3915. 